\title{
Asymmetric Henry Reaction of Nitromethane with Substituted Aldehydes Catalyzed by Novel In Situ Generated Chiral $\mathrm{Bis}\left(\beta\right.$-Amino Alcohol- $\mathrm{Cu}(\mathrm{OAc})_{2} \cdot \mathrm{H}_{2} \mathrm{O}$ Complex
}

\author{
Abdullah Saleh Alammari, Abdullah Mohammed Al-Majid *, Assem Barakat (D, Saeed Alshahrani, \\ Mohammad Ali and Mohammad Shahidul Islam *(D)
}

check for updates

Citation: Alammari, A.S.; Al-Majid, A.M.; Barakat, A.; Alshahrani, S.; Ali, M.; Islam, M.S. Asymmetric Henry Reaction of Nitromethane with Substituted Aldehydes Catalyzed by Novel In Situ Generated Chiral $\mathrm{Bis}\left(\beta\right.$-Amino Alcohol- $\mathrm{Cu}(\mathrm{OAc})_{2} \cdot \mathrm{H}_{2} \mathrm{O}$ Complex. Catalysts 2021, 11, 1208. https://doi.org/10.3390/catal 11101208

Academic Editor: Takeshi Ohkuma

Received: 29 August 2021

Accepted: 1 October 2021

Published: 8 October 2021

Publisher's Note: MDPI stays neutral with regard to jurisdictional claims in published maps and institutional affiliations.

Copyright: (c) 2021 by the authors. Licensee MDPI, Basel, Switzerland. This article is an open access article distributed under the terms and conditions of the Creative Commons Attribution (CC BY) license (https:// creativecommons.org/licenses/by/ $4.0 /)$.
Department of Chemistry, College of Science, King Saud University, P.O. Box 2455, Riyadh 11451, Saudi Arabia; alamary1401@yahoo.com (A.S.A.); ambarakat@ksu.edu.sa (A.B.); 436106738@student.ksu.edu.sa (S.A.); maly.c@ksu.edu.sa (M.A.)

* Correspondence: amajid@ksu.edu.sa (A.M.A.-M.); mislam@ksu.edu.sa (M.S.I.); Fax: +96-61-1467-5992 (M.S.I.)
Abstract: Novel chiral thiophene-2,5-bis( $\beta$-amino alcohol) ligands (L1-L5) were designed and synthesized from thiophene-2,5-dicarbaldehyde (3) with chiral $\beta$-amino alcohols (4a-e) in 4 steps with overall $23 \%$ yields. An in situ generated $\mathrm{L}-\mathrm{Cu}(\mathrm{OAc})_{2} \cdot \mathrm{H}_{2} \mathrm{O}$ catalyst system was found to be highly capable catalyst for the asymmetric Henry reaction of nitromethane (7) with various substituted aromatic aldehydes $(\mathbf{6 a}-\mathbf{m})$ producing chiral nitroaldols product $(\mathbf{8 a}-\mathbf{m})$ with excellent enantiomeric purity (up to $94.6 \%$ ee) and up to $>99 \%$ chemical yields. $20 \mathrm{~mol} \%$ of $\mathbf{L} 4-\mathrm{Cu}(\mathrm{OAc})_{2}$ catalyst complex in $\mathrm{EtOH}$ was effective for the asymmetric Henry transformation in $24 \mathrm{~h}$, at ambient temperature. Ease of ligand synthesis, use of green solvent, base free reaction, mild reaction conditions, high yields and excellent enantioselectivity are all key factors that make this catalytic system robust and highly desirable for the access of versatile building block $\beta$-nitro alcohol in practical catalytic usage via asymmetric Henry reaction.

Keywords: asymmetric catalysis; Henry reaction; Lewis acid; amino alcohols; chiral thiophene-2,5bis-( $\beta$-amino alcohol) ligands

\section{Introduction}

The catalytic synthesis of chiral building blocks is highly desirable to many researchers worldwide because enantiomeric enriched molecules have numerous medicinal importance and applications [1-5]. In recent years, significant improvement has been made in the development of newly synthesized and designed stereoselective catalytic systems in order to access enantiomerically enriched molecules $[2,6,7]$.

Lewis-acid metal catalyzed nucleophilic addition of nitroalkane to carbonyl compound is an important aspect allowing us to furnace a large number of important molecular frameworks [8,9]. Henry reaction [10] (i.e., nitroaldol condensation) is one of the prominent transformations to access a wide range of strategically fundamental molecular structures such as $\beta$-hydroxy nitro alkanes, $\alpha$-hydroxy carboxylic acids and 1,2-amino-alcohols, etc., in a forthright fashion $[9,11]$. Henry reaction also provides a facile and direct access of various versatile building block $\beta$-nitro alcohols $[10,12,13]$ which are the vital skeleton found in many biologically active compounds, such as antibiotics L-acosamine [14], anti-asthmatic drug $(R)$-salmeterol [15], fungicide (S)-spirobrassinin [16] and bestatin [17]. Due to the dual functionality of $\beta$-nitro alcohol, it can be easily transformed into various functionalities via a several roots such as reduction of nitro group into amine, dehydration leads to nitro-olefin, denitration, or other transformations such as Nef reaction and retro-Henry reaction (Figure 1) [18]. Asymmetric Henry approach has been widely reported in the literature; for example, Suami and coworkers employed the asymmetric Henry reaction as a key step during the total synthesis of nucleoside antibiotics 'tunicamycin- $\mathrm{V}$ ' $[12,19]$. 
The total synthesis of tetrodotoxin from D-glucose, using two-step Henry reactions was developed by Sato group [20]. Of late, Dixon et al. reported an efficient process for the total synthesis of natural products marine alkaloid 'manzamine $A^{\prime}$ ', and '(-)-nakadomarin $\mathrm{A}^{\prime}$ involving aza-Henry and Henry reaction [21,22] (Figure 2). It is evident from the above facts that we should emphasize the great utility of the Henry reaction in practical organic transformation.

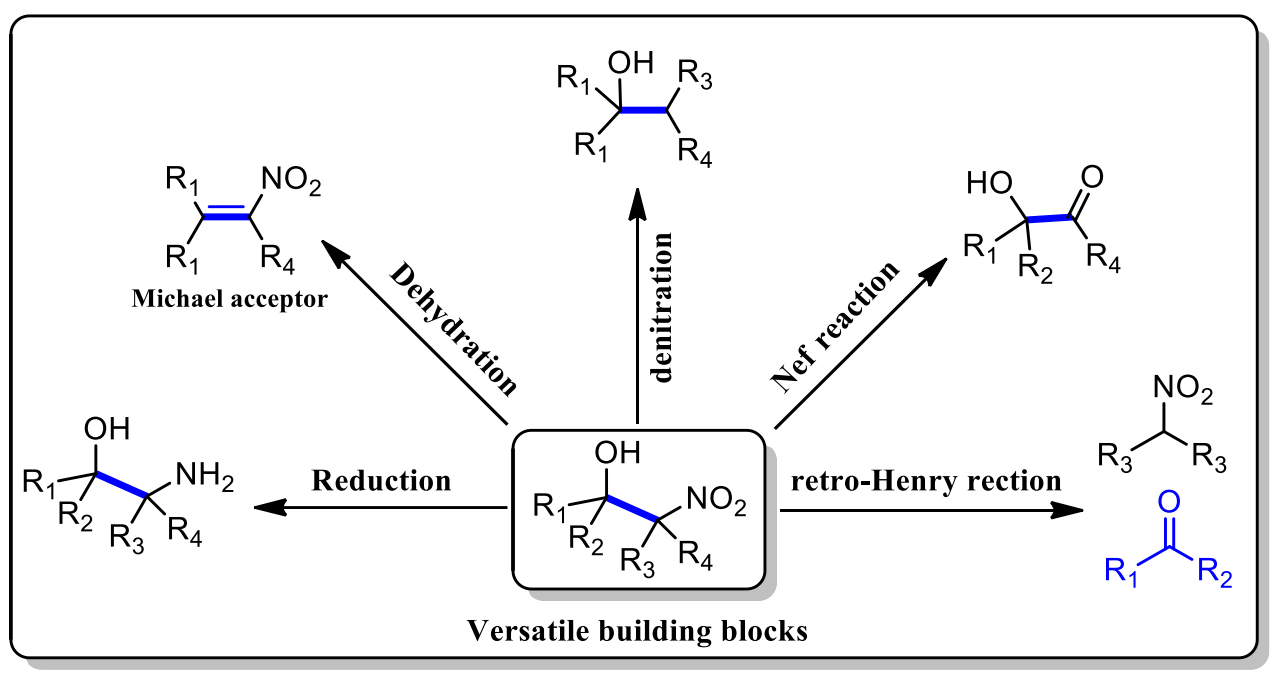

Figure 1. Versatile building blocks can be formulated from Henry-aldol products.

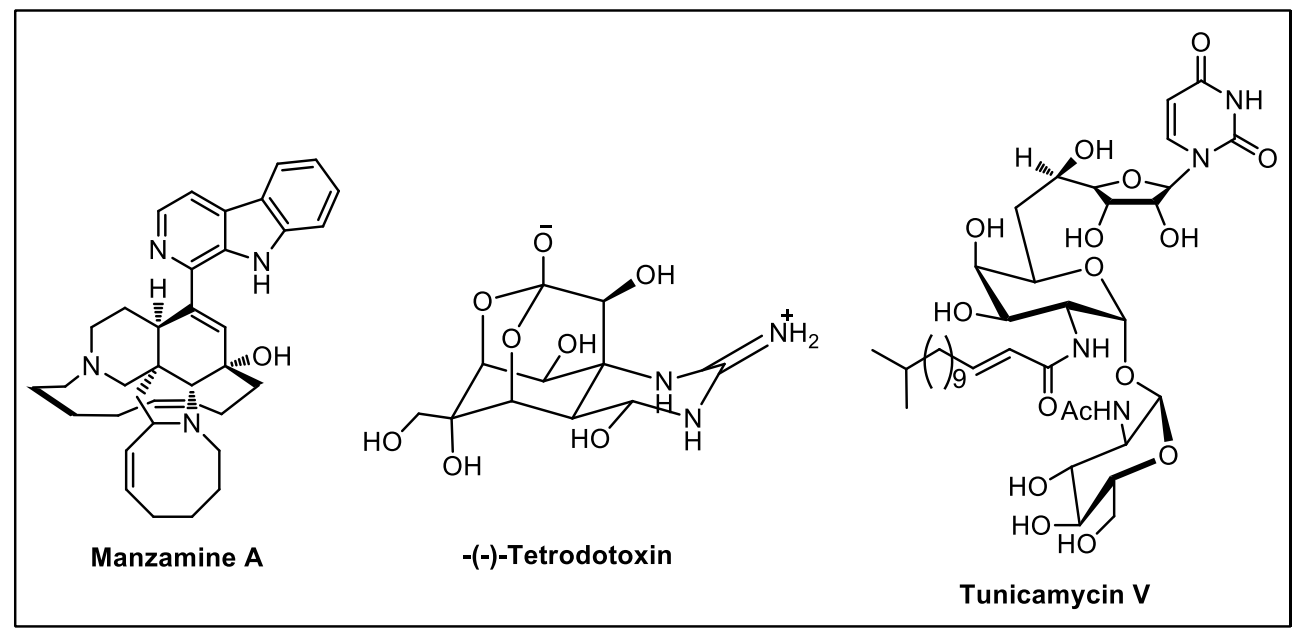

Figure 2. The application of Henry reaction in natural products.

The catalytic-controlled enantioselective Henry reaction was developed and reported for the first time by Shibasaki [23]. Since then, significant efforts have been made for the development of an efficient catalytic system including both metal catalysts [24], as well as organocatalysts [25]. For example, copper(II) complexes of chiral ethane-1,2-diamine derivatives [26,27], bis(oxazolidine)ligands-copper(II) complexes [28], 1,10-binaphthalene2,20-diol based lanthanide complexes [29], and $C_{2}$-symmetric alcohols induced dinuclear zinc(II) complexes [30] were used as a metal-based catalyst in asymmetric Henry reaction applied successfully, while chiral cinchona alkaloids [31], bis(thioureas) [32,33] and guanidines [34] were explored as examples of organocatalysts for Henry reaction which have proved to be work efficiently.

Several enantioselective Henry reactions have been documented recently, such as application of chiral trans-cyclohexane-1,2-diamine-copper complexes described in the asymmetric Henry reaction in modest to excellent enantioselectivity [35-42]. Formation 
of binuclear copper(II) complexes of two chiral $\beta$-amino alcohols connected to benzene ring in 1,4-positions to the central backbone developed and explored in asymmetric Henry reaction by Zhang et al. [43]. Moreover, transition metal catalyzed asymmetric Henry reactions summarized by Velmathi et al. prior to 2011 have been well documented [33,44-49]. Many reports suggested that the chiral bi-functional coordination complexes system efficiently controls the stereochemical outcomes of the reaction (Shibasaki [23], Jørgensen [50], Trost [30], Yamada [51] and Palomo [52]). However, in their findings some genuine limitations have been found, such as use of organic bases like nucleophilic silyl nitronates as additives, low reaction temperatures (even lower than $-20^{\circ} \mathrm{C}$ ) and comparatively high catalyst loadings, etc. Evans and coworkers have developed a catalytic system based on copper acetate-chiral bis-oxazoline ligand for Henry reaction affording nitroaldol in high yield with excellent enantioselectivity [28]. In general, readily available, cheap, effective catalytic system, mild reaction conditions, low catalyst loading, and a high degree of stereo-induction are still a challenging task for the development of sustainable catalytic systems [53]. Therefore, design, synthesis and development of new chiral ligand based on an optically active system is still desirable for the catalytic asymmetric Henry reactions [43].

Since the main family of successful chiral ligands predominantly belongs to diphosphine, diamine, di-ol etc, i.e., phosphorous, nitrogen and oxygen-containing substrate, large amount of work has been done on these areas. From the past few years, researchers have been eagerly keen to develop chiral ligands for enantioselective catalysis, based on sulfur containing compounds due to high coordination ability of sulfur atom to most of the transition metals. The sulfur atom is considered as a soft atom which can form strong bonds with soft metals like $\mathrm{Cu}(\mathrm{II})$. In addition, sulfur ligands are poor $\sigma$-donor and poor $\pi$-acceptor ligands as compared to phosphine ligands which results in strong metal-sulfur bond strength. Moreover, sulfur-containing compounds are easily accessible and easy to handle as well as store due to their more tolerance to air as compared to phosphine containing ligands, and therefore they are highly stable [54].

In this article, we report the synthesis of chiral ligands based on thiophene framework and their applications in asymmetric henry reaction as part of our ongoing research project in our laboratory.

Based on these facts, we synthesized a new chiral thiophene-2,5-bis ( $\beta$-amino alcohol) ligands (L1-L5) (Scheme 1) possessing two trans- $\beta$-hydroxy amine units attached to C1 and $\mathrm{C} 4$ carbon of thiophene ring and we explored their utilities for asymmetric Henry reaction (nitroaldol condensation).

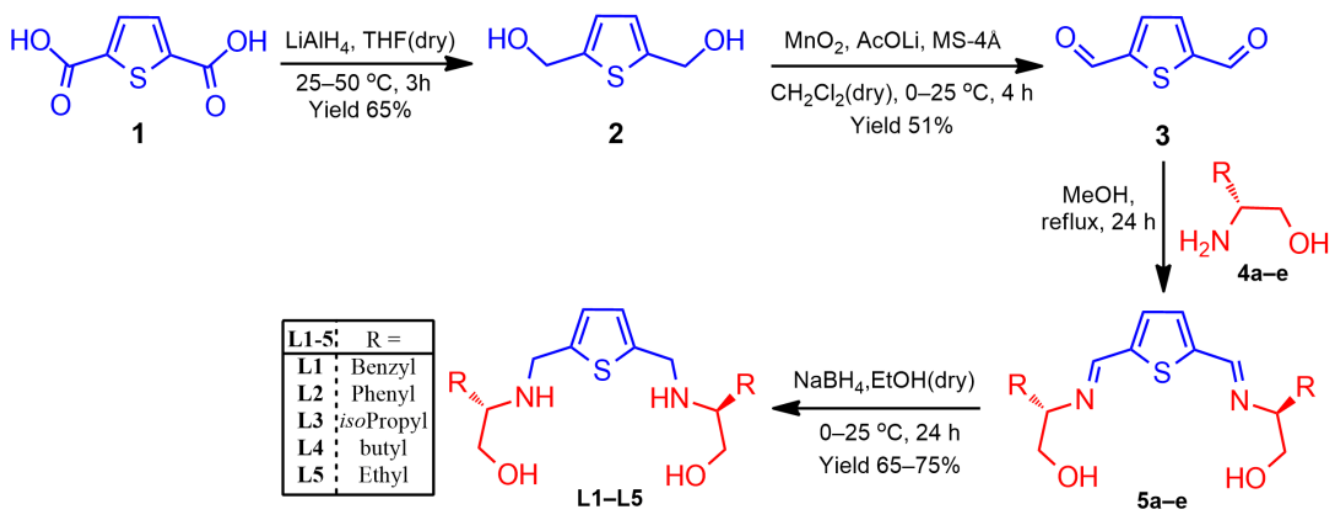

Scheme 1. Synthesis of New amino alcohol chiral Ligands (L1-L5). 


\section{Results and Discussion}

\subsection{Synthesis of Ligand L1-L5}

A series of $C_{2}$-symmetric thiophene based chiral bis ( $\beta$-amino alcohol) ligands (L1-L5) have been synthesized in 4 steps from a commercially available starting material thiophene2,5-dicarboxlyic acid (1) and variety of chiral $\beta$-amino alcohols (4a-e) with good yield and excellent optical purity as depicted in Scheme 1. Initially, thiophene-2,5-dicarboxlyic acid (1) was reduced into thiophene-2,5-diyldimethanol (2) by 2.5 eq. of $\mathrm{LiAlH}_{4}$ in dry THF with $65 \%$ chemical yield, followed by reported literature procedure $[55,56]$. The product thiophene-2,5-dicarbaldehyde (3) was obtained as dark red solid in 51\% isolated yield from the oxidation of thiophene-2,5-diyldimethanol (2) using the mixture of $\mathrm{MnO}_{2}$ (2.2 eq.) and Lithium acetate (2.2 eq.) in dry $\mathrm{CH}_{2} \mathrm{Cl}_{2}$ as oxidizing agent with $30 \mathrm{mg}$ pre-treated $4 \AA$ molecular sieve as reported in the literature $[57,58]$. Thiophene-2,5-dicarbaldehyde (3) was then allowed to react with various chiral $\beta$-amino alcohols ( 2.2 eq. $4 \mathbf{a}-\mathbf{e}$ ) in methanol under reflux for $24 \mathrm{~h}$ afforded the corresponding enamines (4a-e) those were successfully reduced by $\mathrm{NaBH}_{4}$ (2.6 eq.) [59], in ethanol to yield the desired chiral bis ( $\beta$-amino alcohol) ligands (L1-L5) in 65-75\% isolated yield and excellent optical purity (Scheme 1). All the intermediates $(\mathbf{2}, \mathbf{3}, \mathbf{5 a}-\mathbf{e})$ and final ligands (L1-L5) were characterized by NMR, LCMS and FT-IR techniques.

\subsection{Catalytic Studies of the Henry Reaction}

At the very outset, in order to achieve isolated crystalline material of the metal-ligand complex, several metal salts including $\left(\mathrm{Cu}(\mathrm{OAc})_{2} \cdot \mathrm{H}_{2} \mathrm{O}, \mathrm{CuBr}_{2}, \mathrm{CuCl}_{2}, \mathrm{Cu}(\mathrm{OTf})_{2}, \mathrm{Zn}(\mathrm{OTf})_{2}\right)$ were allowed to react with pure ligands (L1-L5) in various solvents (ethanol, methanol, toluene, diethylether, and THF) under inert atmosphere using Schlenk tube technique. Despite multiple attempts were carried out, but the desired ligand metal complexes were unsuccessfully isolated. Therefore, we decided to test the catalytic activity of our ligand in situ generated complex with metal salt. Initially, we tested the efficiency of our newly synthesized ligands (L1-L5) for asymmetric Henry reaction (Scheme 2) in the presence of $\mathrm{Cu}(\mathrm{OAc})_{2} \cdot \mathrm{H}_{2} \mathrm{O}$ in ethanol by choosing nitromethane (7) and 2-nitrobenzaldehyde (6a) as model substrate (Table 1). The first attempt, equimolar of ligands (L1-L5) and $\mathrm{Cu}(\mathrm{OAc})_{2} \cdot \mathrm{H}_{2} \mathrm{O}(20 \mathrm{~mol} \%)$ in ethanol $(2 \mathrm{~mL})$ were stirred at $25^{\circ} \mathrm{C}$ under inert atmosphere for $2 \mathrm{~h}$ to generate blue colored solution of $\mathrm{L}-\mathrm{Cu}(\mathrm{OAc})_{2} \cdot \mathrm{H}_{2} \mathrm{O}$ complex followed by addition of the model substrate 2-nitrobenzaldehyde (6a). After 20 min of stirring at room temperature nitromethane (7) was added to the reaction and further stirred for $24-48 \mathrm{~h}$ at ambient temperature to produce nitroaldol Henry product (8a) and the results were summarized in Table 1. Surprisingly, our initial results exhibits that all the ligands (L1-L5) under the above reaction parameters performed very well to induce excellent enantioselectivity (89.9-94.6\% ee) with high chemical yields (90-99\%) (Table 1, entries 1-5). However, Ligand $\mathbf{L} \mathbf{4}$ was found to be the best choice for the asymmetric Henry reaction which yielded $99 \%$ chemical yield and high enantioselectivity $94.6 \%$ ee in 24 h (Table 1, entry 4 ) at ambient temperature. In order to optimize the catalyst loading, we further perform the Henry reaction by employing the most efficacy catalyst system i.e., $\mathrm{L} 4-\mathrm{Cu}(\mathrm{OAc})_{2} \cdot \mathrm{H}_{2} \mathrm{O}$ complex under different catalyst loading $(5,10,25 \mathrm{~mol} \%)$ in ethanol for $24 \mathrm{~h}$ but unfortunately no further improvements were observed (Table 1, entries 7-9). To achieve higher enantioselectivity, the Henry reaction was carried out at lower temperature $10{ }^{\circ} \mathrm{C}$ for $48 \mathrm{~h}$ which produces $87 \%$ chemical yields and $90.4 \%$ ee (Table 1 , entries 6 ). It was obvious that $20 \mathrm{~mol} \%$ of the catalytic system $\mathbf{L} 4-\mathrm{Cu}(\mathrm{OAc})_{2} \cdot \mathrm{H}_{2} \mathrm{O}$ in ethanol at $25^{\circ} \mathrm{C}$ for $24 \mathrm{~h}$ was found to be the best catalytic system for asymmetric Henry reaction. 


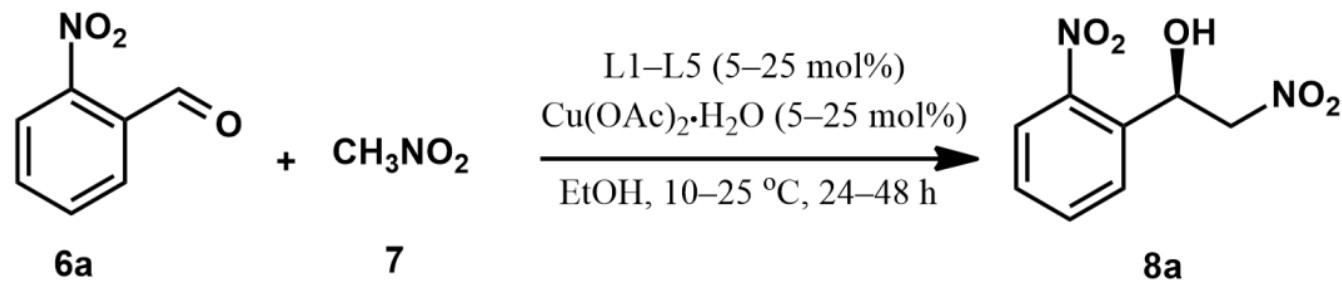

Scheme 2. Asymmetric Henry Reaction of nitromethane (7) with 2-nitrobenzaldehyde (6a), ligands screening.

Table 1. Henry reaction investigation of nitromethane (7) with 2-nitrobenzaldehyde (6a) as model substrate; Ligand screening.

\begin{tabular}{|c|c|c|c|c|c|c|}
\hline Entry [a] & Ligands & $\begin{array}{c}\mathrm{L}: \mathrm{Cu}(\mathrm{OAc})_{2} \cdot \mathrm{H}_{2} \mathrm{O} \\
(\mathrm{mol} \%)\end{array}$ & Temp $[t]$ & $\begin{array}{l}\text { Time } \\
{[T / h]}\end{array}$ & Yield (\%) ${ }^{[b]}$ & ee $\%[c]$ \\
\hline 1. & L1 & 20 & $25^{\circ} \mathrm{C}$ & 24 & 99 & 92.3 \\
\hline 2. & $\mathrm{~L} 2$ & 20 & $25^{\circ} \mathrm{C}$ & 24 & 90 & 94.0 \\
\hline 3. & L3 & 20 & $25^{\circ} \mathrm{C}$ & 48 & 91 & 89.9 \\
\hline 4. & $\mathrm{~L} 4$ & 20 & $25^{\circ} \mathrm{C}$ & 24 & 99 & 94.6 \\
\hline 5. & L5 & 20 & $25^{\circ} \mathrm{C}$ & 48 & 89 & 90.0 \\
\hline 6. & L4 & 20 & $10^{\circ} \mathrm{C}$ & 48 & 87 & 90.4 \\
\hline 7. & L4 & 5 & $25^{\circ} \mathrm{C}$ & 24 & 80 & 87.1 \\
\hline 8. & L4 & 10 & $25^{\circ} \mathrm{C}$ & 24 & 88 & 85.2 \\
\hline 9. & $\mathrm{~L} 4$ & 25 & $25^{\circ} \mathrm{C}$ & 24 & 99 & 94.0 \\
\hline
\end{tabular}

${ }^{[a]}$ Reaction was performed on a $0.2 \mathrm{mmol}$ scale of aldehyde and $2 \mathrm{mmol}$ of nitromethane; ${ }^{[\mathrm{b}]}$ Yield of the isolated product after flash column chromatography; ${ }^{[c]}$ Determined by HPLC analysis on a Daicel Chiralcel OD-H column $(25 \mathrm{~cm} \times 4.6 \mathrm{~mm} \times 5 \mu \mathrm{m})$.

Next, aiming to find out the best medium for Henry reaction (Scheme 3), several solvents were examined like methanol, isopropanol, isobutanol, and tetrahydrofuran using best reaction condition $20 \mathrm{~mol} \% \mathrm{~L} 4-\mathrm{Cu}(\mathrm{OAc})_{2} \cdot \mathrm{H}_{2} \mathrm{O}$ at room temperature for $24-48 \mathrm{~h}$ and the results are shown in Table 2. Almost in all solvent's reaction proceeded very well with commendable yields (79-99\%) and enantioselectivity (68.3-86.6\% ee) (Table 2, entries 1-4). However, in methanol high yield (88\%) and high enantioselectivity (85.3\% ee) were observed, on the contrary in THF lowest yield (79\%) and lowest enantiomeric excess ( $68.3 \%$ ee) was obtained (Table 2, entries $1 \& 4)$. Moderate to excellent yield $(80 \%$ \& $99 \%)$ and enantiomeric excess $(86.6 \%, 82.6 \%$ ee) were achieved when the reaction was performed in isopropanol, isobutanol respectively (Table 2, entries $2 \& 3$ ). However, ethanol remains the best choice for the asymmetric Henry reaction.<smiles>O=Cc1ccccc1[N+](=O)[O-]</smiles>

$6 a$<smiles>CO[N+](=O)[O-]</smiles>
7

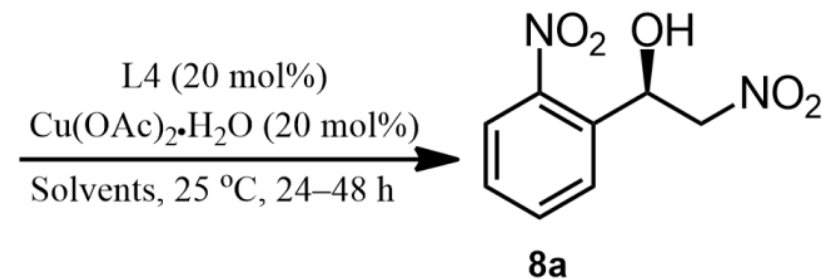

Scheme 3. Asymmetric Henry Reaction of nitromethane (7) with 2-nitrobenzaldehyde (6a), solvent screening. 
Table 2. Solvent screening on the enantioselective Henry Reaction of nitromethane (7) with 2nitrobenzaldehyde (6a).

\begin{tabular}{|c|c|c|c|c|}
\hline Entry ${ }^{[a]}$ & Solvent & Time (h) & Yield (\%) ${ }^{[b]}$ & ee $\%$ [c] \\
\hline 1. & $\mathrm{MeOH}$ & 24 & 88 & 85.3 \\
\hline 2. & $i-\mathrm{PrOH}$ & 48 & 80 & 86.6 \\
\hline 3. & $t-\mathrm{BuOH}$ & 48 & 99 & 82.6 \\
\hline 4. & THF & 48 & 79 & 68.3 \\
\hline
\end{tabular}

[a] Reaction was performed on a $0.2 \mathrm{mmol}$ scale of aldehyde and $2 \mathrm{mmol}$ of nitromethane; ${ }^{[\mathrm{b}]}$ Yield of the isolated product after flash column chromatography; ${ }^{[c]}$ Determined by HPLC analysis on a Daicel Chiralcel OD-H column $(25 \mathrm{~cm} \times 4.6 \mathrm{~mm} \times 5 \mu \mathrm{m})$.

Further, we investigated the effects of various metal salts such as $\mathrm{Cu}(\mathrm{OAc})_{2} \cdot \mathrm{nH}_{2} \mathrm{O}$, $\mathrm{Zn}(\mathrm{OTf})_{2}, \mathrm{Cu}(\mathrm{OTf})_{2}, \mathrm{CuBr}_{2}, \mathrm{CuCl}_{2}$ and $\mathrm{Zn}(\mathrm{OAc})_{2} \cdot 2 \mathrm{H}_{2} \mathrm{O}$ as a Lewis acid with keeping in mind that the other reaction parameters unchanged with prolonged reaction time up to $72 \mathrm{~h}$ (Scheme 4), the results are summarized in Table 3. From these results it can be infer that the ligand $\mathbf{L} 4$ with $\mathrm{Cu}(\mathrm{II})$ acetate complex is the only choice for the asymmetric Henry reaction which is capable of inducing chirality (up to $94.6 \%$ ) into the nitroaldol product 8a in high chemical yield (Table 3, entry 1). The best performance of copper(II) acetate perhaps could be attributed to its high effectiveness in chelate formation with ligands than the other tested copper(II) salts [60]. Although L4-Zn(OTf $)_{2}$ system produced excellent chemical yield but failed to induce enantioselectivity (only 6.6\% ee) (Table 3, entry 3 ), while L4- $\mathrm{Zn}(\mathrm{OAc})_{2} \cdot 2 \mathrm{H}_{2} \mathrm{O}$ complex produce $50 \%$ yield with $19 \%$ ee (Table 3 , entry 7 ) which is obviously insignificant. The metal salts $\mathrm{Cu}(\mathrm{OTf})_{2}, \mathrm{CuBr}_{2}$ and $\mathrm{CuCl}_{2}$ in combination with L4 were found to inactive in catalyzing the Henry reaction (Table 2, entries 4-6).

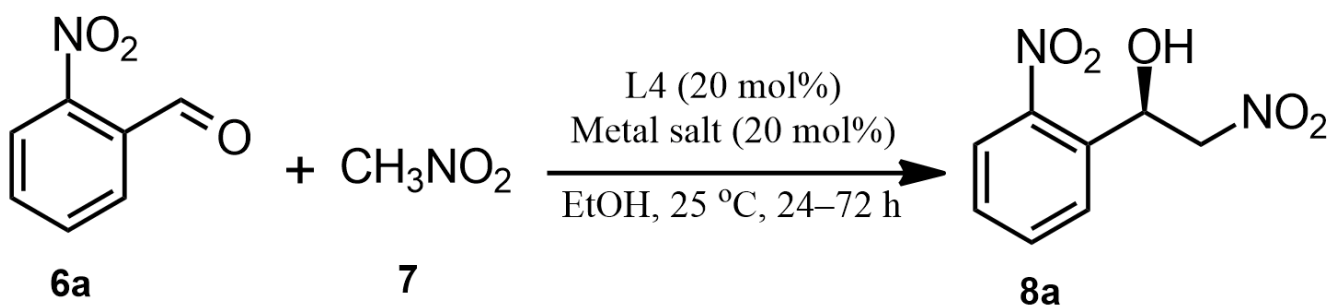

Scheme 4. Asymmetric Henry Reaction of nitromethane (7) with 2-nitrobenzaldehyde (6a), metal salts screening.

Table 3. Effect of metal salt on the enantioselective Henry Reaction of nitromethane with 2nitrobenzaldehyde.

\begin{tabular}{|c|c|c|c|c|}
\hline Entry $^{[a]}$ & Metal Salt & Time $[\mathrm{T} / \mathrm{h}]$ & Yield (\%) ${ }^{[b]}$ & ee $(\%)$ [c] \\
\hline 1. & $\mathrm{Cu}(\mathrm{OAc})_{2} \cdot \mathrm{H}_{2} \mathrm{O}$ & 24 & 99 & 94.6 \\
\hline 2. & $\mathrm{Cu}(\mathrm{OAc})_{2} \cdot \mathrm{nH}_{2} \mathrm{O}$ & 24 & 97 & 94.3 \\
\hline 3. & $\mathrm{Zn}(\mathrm{OTf})_{2}$ & 48 & 99 & 6.6 \\
\hline 4. & $\mathrm{Cu}(\mathrm{OTf})_{2}$ & 72 & 20 & 1 \\
\hline 5. & $\mathrm{CuBr}_{2}$ & 72 & - & - \\
\hline 6. & $\mathrm{CuCl}_{2}$ & 72 & - & - \\
\hline 7. & $\mathrm{Zn}(\mathrm{OAc})_{2} \cdot 2 \mathrm{H}_{2} \mathrm{O}$ & 72 & 50 & 19.0 \\
\hline
\end{tabular}

[a] Reaction was performed on a $0.2 \mathrm{mmol}$ scale of aldehyde and $2 \mathrm{mmol}$ of nitromethane; ${ }^{[\mathrm{b}]}$ Yield of the isolated product after flash column chromatography; ${ }^{[c]}$ Determined by HPLC analysis on a Daicel Chiralcel OD-H column $(25 \mathrm{~cm} \times 4.6 \mathrm{~mm} \times 5 \mu \mathrm{m})$.

To illustrate the generality of this catalytic approach, asymmetric Henry reaction (Scheme 5) was performed with a variety of aromatic aldehydes (6a-m, 13 examples) and nitromethane (7) utilizing the optimized reaction conditions $\left(20 \mathrm{~mol} \% \mathbf{L} 4-\mathrm{Cu}(\mathrm{OAc})_{2} \cdot \mathrm{H}_{2} \mathrm{O}\right.$ in ethanol for $24-48 \mathrm{~h}$ at rt). All of the screened aromatic aldehydes produced corresponding nitroaldol product $(\mathbf{8} \mathbf{a}-\mathbf{m})$ predominantly with enriched of $(R)$-enantiomer with moder- 
ate to excellent isolated yields (66-99\%) and enantioselectivities (53-95\% ee) (Scheme 5, Table 4). Although, higher enantioselectivities (81-94\% ee) and yields (82-99\%) were found corresponding to the ortho, meta, para nitro-benzaldehyde, $p$-tolualdehyde and benzaldehyde (Table 4, entry 1-3, 11, 13), However, there is no clear, conclusive evidence which suggests that there is any kind of influence in the enantioselectivity due to steric factor as well as electronic environments of the substituents in the aromatic ring (Table 4, entries 1-13). Previously asymmetric Henry reaction has been reported by Lu et al. [59] with very good yield (89-98\% ee) in $24-48 \mathrm{~h}$ at $10^{\circ} \mathrm{C}$, using similar type of amino alcohol chiral ligand- $\mathrm{Cu}(\mathrm{OAc})_{2} \cdot \mathrm{H}_{2} \mathrm{O}$. Jin et al. reported asymmetric Henry reaction with excellent enantiomeric excess using $\mathrm{CuBr}$-diamine catalyst in the presence of another additive like pyridine [40]. Amino Alcohol-Cu(II) complex has been used to catalyze asymmetric Henry reaction by Qin et al. and very high ee \% obtained with prolonged time 48-72 h. However, in our study we have optimized the reaction at room temperature in ethanol as green solvent, good to high enantiomeric access were achieved without using any additives within reasonable timeframe (24-48 h) at ambient temperature. Several Literature suggest that the retention time of the $R$ enantiomer is lesser than the $S$ enantiomer for the nitroaldol products. Therefore, the absolute configuration of all the newly synthesized chiral nitroaldol product $(\mathbf{8} \mathbf{a}-\mathbf{m})$ were assigned as $R$ respectively by comparing their retention time found in reported literature $[40,59,61,62]$.

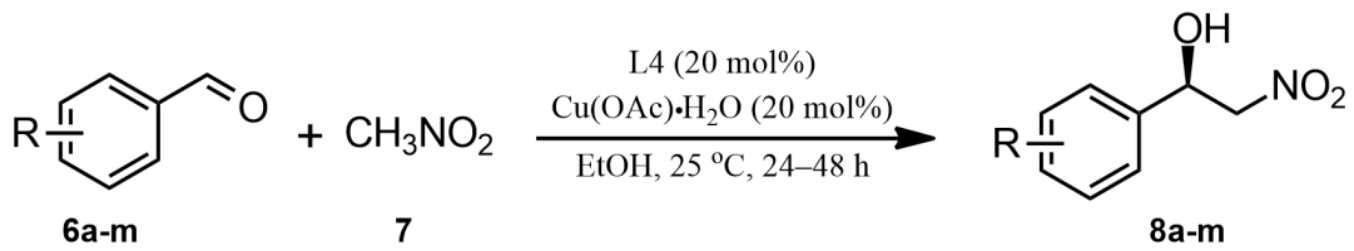

Scheme 5. Asymmetric Henry Reaction of nitromethane substrate scope.

Table 4. Substrate scope of aldehydes and nitromethane in the asymmetric Henry reaction.

\begin{tabular}{lccccc}
\hline \multicolumn{1}{c}{ Entry ${ }^{[\mathbf{a}]}$} & $\mathbf{R}(\mathbf{6 a}-\mathbf{m})$ & Products & Time (h) & Yield (\%) & ee $\mathbf{\text { [b] }}$ [c] \\
\hline 1. & $2-\mathrm{NO}_{2}$ & $\mathbf{8 a}$ & 24 & 99 & $94.6(R)$ \\
2. & $4-\mathrm{NO}_{2}$ & $\mathbf{8 b}$ & 24 & 96 & $81.3(R)$ \\
3. & $3-\mathrm{NO}_{2}$ & $\mathbf{8 c}$ & 48 & 91 & $81.2(R)$ \\
4. & $4-\mathrm{Br}$ & $\mathbf{8 d}$ & 48 & 80 & $76.4(R)$ \\
5. & $\mathrm{Naph}$ & $\mathbf{8 e}$ & 48 & 66 & $75.1(R)$ \\
6. & $4-\mathrm{CF}_{3}$ & $\mathbf{8 f}$ & 24 & 82 & $58.9(R)$ \\
7. & $2,4-\mathrm{Cl}$ & $\mathbf{8 g}$ & 48 & 86 & $53.0(R)$ \\
8. & $4-\mathrm{Cl}$ & $\mathbf{8 h}$ & 48 & 66 & $73.1(R)$ \\
9. & $4-\mathrm{F}$ & $\mathbf{8 i}$ & 48 & 75 & $60.9(R)[\mathrm{d}]$ \\
10. & $4-\mathrm{OCH}_{3}$ & $\mathbf{8 j}$ & 24 & 77 & $63.9(R)[\mathrm{d}]$ \\
11. & $4-\mathrm{CH}_{3}$ & $\mathbf{8 k}$ & 24 & 85 & $81.3(R)$ \\
12. & $3-\mathrm{CH}_{3}$ & $\mathbf{8 1}$ & 48 & 88 & $60.8(R)[\mathrm{d}]$ \\
13. & $\mathrm{H}$ & $\mathbf{8 m}$ & 48 & 82 & $89.2(R)$
\end{tabular}

[a] Reactions were performed with the aldehyde $(0.2 \mathrm{mmol})$ and nitromethane $(1 \mathrm{mmol})$ in $2 \mathrm{~mL}$ of ethanol; [b] Isolated yield after column chromatography; ${ }^{\text {[c] }}$ Determined by chiral HPLC analysis on a Daicel Chiralcel OD-H column $(25 \mathrm{~cm} \times 4.6 \mathrm{~mm} \times 5 \mu \mathrm{m})$. ${ }^{\text {dd] }}$ Due to some impurities altered the ee $\%$.

\section{Experiments}

\subsection{General}

Reagents obtained from commercial suppliers were used without further purification. Preparation of bis( $\beta$-amino alcohol) ligands was performed in flask dried glassware under a static pressure of nitrogen. Solvents were dried prior to use following standard procedures. Reactions were monitored by thin layer chromatography using Merck (silica gel 60 Kieselgel F254 TLC (Merck, Kenilworth, NJ, USA) and column chromatography was performed on silica gel 100-200 mesh (40-63 $\mu \mathrm{m}, 200$ mesh, ASTM) from Merck using the indicated 
solvents. ${ }^{1} \mathrm{H}$ and ${ }^{13} \mathrm{C}-\mathrm{NMR}$ spectra were recorded in $\mathrm{CDCl}_{3}$ and DMSO- $d_{6}$ on a Jeol Spectrometer (Jeol, Tokyo, Japan) (400 MHz and $500 \mathrm{MHz})$. The chemical shifts are reported in ppm relative to $\mathrm{CDCl}_{3}(\delta(\mathrm{ppm})=7.26)$ or $d_{6}$-DMSO $(\delta(\mathrm{ppm})=2.50)$ for ${ }^{1} \mathrm{H}-\mathrm{NMR}$. For the ${ }^{13} \mathrm{C}-\mathrm{NMR}$ spectra, the residual $\mathrm{CDCl}_{3}(\delta(\mathrm{ppm})=77.16$ in $\mathrm{ppm})$ or $d_{6}$-DMSO $(\delta(\mathrm{ppm})=39.5$ in $\mathrm{ppm}$ ). All the racemic products were freshly prepared as per the method reported in the literature [61]. Infrared spectra were recorded on a Thermo Scientific Nicolet iS10 FT-IR spectrometer (Thermo Fisher Scientific, Waltham, MA, USA). Enantiomeric ratios were determined by analytical chiral HPLC analysis on a Shimadzu LC-20A Prominence instrument (Shimadzu, Kuoto, Japan) with a chiral stationary phase using Daicel Chiralcel OD-H columns (Chiral Technologies Europe, Illkirch Graffenstaden, France) (80-95\% nhexane/iso-propanol) (Supplementary Materials). Optical rotations were obtained with a Perkin-Elmer 343 polarimeter (Perkin-Elmer, Waltham, MA, USA). Melting points (m.p.) were recorded on a Thomas-Hoover (Thomas-Hoover, Keller, TX, USA) capillary melting point apparatus and were not corrected. Mass spectrometric analysis was done using ESI mode on AGILENT Technologies 6410-triple quad LC/MS instrument (Agilent, Santa Clara, CA, USA). Elemental analyses were performed on Perkin-Elmer PE 2400 CHN Elemental Analyzer with autosampler, $\mathrm{CHN}$ mode.

\subsection{Synthesis of thiophene-2,5-diyldimethanol (2)}

A solution of thiophene-2,5-dicarboxylic acid $(1.40 \mathrm{~g}, 8.14 \mathrm{mmol})$ in dry THF $(50 \mathrm{~mL})$ were added slowly to a the pre stirred suspension of lithium aluminum hydride $(0.76 \mathrm{~g}$, $20.00 \mathrm{mmol})$ in dry tetrahydrofuran $(100 \mathrm{~mL})$ at $0^{\circ} \mathrm{C}[55,56]$. The reaction was then warmed up to $50^{\circ} \mathrm{C}$ and stirred for $3 \mathrm{~h}$ followed by cooling of temperature to ambient temperature. The reaction was then neutralized with saturated $\mathrm{Na}_{2} \mathrm{SO}_{4}$ solution and filtered through celite bed. The filtrate was concentrated in vacuum to afford thiophene-2,5-diyldimethanol (2) was obtained as red oil $(0.76 \mathrm{~g}, 65 \%)$; $\mathrm{IR}\left(\mathrm{KBr}, \mathrm{cm}^{-1}\right)$ : 3364, 3082, 3066, 2956, 1613, 1543, $1515,1464,1023,741 ;{ }^{1} \mathrm{H}-\mathrm{NMR}\left(500 \mathrm{MHz}, \mathrm{CDCl}_{3}\right): \delta(\mathrm{ppm})=6.86(\mathrm{~s}, 2 \mathrm{H}$, thiophene-H), 4.78 $\left(\mathrm{s}, 4 \mathrm{H}, \mathrm{CH}_{2}\right), 2.00(\mathrm{~s}, 2 \mathrm{H}, \mathrm{OH}) ;{ }^{13} \mathrm{C}-\mathrm{NMR}\left(126 \mathrm{MHz}, \mathrm{CDCl}_{3}\right) \delta(\mathrm{ppm})=144.46,125.42,60.33$; $\mathrm{LC} / \mathrm{MS}$ (ESI, $\mathrm{m} / \mathrm{z}$ ): found $145.02[\mathrm{M}+\mathrm{H}]^{+}$, exact mass 144.02 for $\mathrm{C}_{6} \mathrm{H}_{8} \mathrm{O}_{2} \mathrm{~S}$; Anal. calcd. for $\mathrm{C}_{6} \mathrm{H}_{8} \mathrm{O}_{2} \mathrm{~S}: \mathrm{C}, 49.98 ; \mathrm{H}, 5.59$ found $\mathrm{C}, 49.73 ; \mathrm{H}, 5.44$.

\subsection{Synthesis of thiophene-2,5-dicarbaldehyde (3)}

A suspension solution of thiophene-2,5-diyldimethanol (2) (144 mg, $1 \mathrm{mmol})$ in dry $\mathrm{CH}_{2} \mathrm{Cl}_{2}(35 \mathrm{~mL})$ were added to a previously stirred suspension solution of $\mathrm{MnO}_{2}$ (191.25 mg, $2.2 \mathrm{mmol})$, Lithium acetate (145.18 mg, $2.2 \mathrm{mmol}$ ) and pre-activated molecular sieve $4 \AA$ ( $30 \mathrm{mg})$ in dry $\mathrm{CH}_{2} \mathrm{Cl}_{2}(20 \mathrm{~mL})$ at room temperature. After $4 \mathrm{~h}$, the reaction turned into dark red suspension which was then filtered through grit-glass filter with Celite 521. The solvents were then removed under reduced pressure to afford crude product. The crude material was purified by column chromatography using silica gel (100-200 mesh) and ethyl acetate $/ n$-hexane ( $40 \%)$ as an eluent to obtain the pure compound (3) as dark red solid (yield 71.5mg, 51\%) [57,58]; m.p. 114- $115^{\circ} \mathrm{C}$; IR (KBr, cm $\left.{ }^{-1}\right)$ : 3083, 3075, 2942, 1721, $1541,1511,1461,1033,741 \mathrm{~cm}^{-1} .{ }^{1} \mathrm{H}-\mathrm{NMR}\left(500 \mathrm{MHz}, \mathrm{CDCl}_{3}\right): \delta(\mathrm{ppm})=10.02(\mathrm{~s}, 2 \mathrm{H}, \mathrm{CHO})$, $7.83\left(\mathrm{~s}, 2 \mathrm{H}\right.$, thiophene-H), $4.76\left(\mathrm{~s}, 4 \mathrm{H}, \mathrm{CH}_{2}\right) .{ }^{13} \mathrm{C}-\mathrm{NMR}\left(126 \mathrm{MHz}, \mathrm{CDCl}_{3}\right) \delta(\mathrm{ppm})=183.58$, 149.25, 135.29; $\mathrm{LC} / \mathrm{MS}$ (ESI, $\mathrm{m} / \mathrm{z}$ ): found $139.99[\mathrm{M}+\mathrm{H}]^{+}$, exact mass for $\mathrm{C}_{6} \mathrm{H}_{8} \mathrm{O}_{2} \mathrm{~S}$ is140.16; Anal. calcd. for $\mathrm{C}_{6} \mathrm{H}_{4} \mathrm{O}_{2} \mathrm{~S}: \mathrm{C}, 51.42 ; \mathrm{H}, 2.88$; found C, 51.22; $\mathrm{H}, 2.94$.

\subsection{General Procedure for Synthesis of Chiral Diamine Alcohol Ligands (L1-L5)}

General Procedure (GP1): In a $100 \mathrm{~mL}$ round bottom flask, thiophene-2,5-dicarbaldehyde (3) $(140 \mathrm{mg} ; 1 \mathrm{mmol})$ and chiral $\beta$-amino alcohol $(4 \mathbf{a}-\mathbf{e})(2.2 \mathrm{mmol})$ were dissolved in dry methanol $(25 \mathrm{~mL})$. The reaction was then vigorously stirred for $24 \mathrm{~h}$ under reflux condition in an inert atmosphere. Then the solvents were evaporated and washed with cold diethyl ether $(2 \times 20 \mathrm{~mL})$ and dried to avail chiral enamine Schiff base $(\mathbf{5 a}-\mathbf{e})$. The chiral enamines $(5 \mathbf{a}-\mathbf{e})(1 \mathrm{mmol})$ suspended in dry ethanol $(20 \mathrm{~mL})$ followed by portion wise addition of $\mathrm{NaBH}_{4}$ (2.6 eq.) in four equal parts and kept on stirring at ambient temperature for $24 \mathrm{~h}$ [59]. 
After completion of the reaction (approximately 24h), solvent was completely removed under reduced pressure and added plenty of water, solid precipitate comes out which was filtrated and further purified by column chromatography using silica gel (100 mesh) and $7-10 \%\left(\mathrm{MeOH} / \mathrm{CH}_{2} \mathrm{Cl}_{2}\right)$ as eluent to obtain the desired Ligands (L1-L5) in good yield (65-75\%).

\subsection{1. ((2S,2'S)-2,2'-((Thiophene-2,5-diylbis(methylene))bis(azanediyl))bis(3-phenylpropan-1-ol) (L1)}

Following GP1, thiophene-2,5-dicarboaldehyde (3) and (S)-2-amino-3-phenylpropan1-ol (4a) were reacted to produce diamine alcohol (L1) as pale brown solid (308 $\mathrm{mg}, 75 \%$ ); m.p. $124-125^{\circ} \mathrm{C} ;[\alpha]_{D}^{25}=+1.39^{\circ}$ (c 0.064, $\left.\mathrm{MeOH}\right) ; \operatorname{IR}\left(\mathrm{KBr}, \mathrm{cm}^{-1}\right)$ : 3390, 3287, 3131, 3058, 2924, $2855,1604,1494,1451,1384,1027,747 \mathrm{~cm}^{-1} .{ }^{1} \mathrm{H}-\mathrm{NMR}\left(500 \mathrm{MHz}, \mathrm{DMSO}-d_{6}\right): \delta(\mathrm{ppm})=7.25$ $(\mathrm{t}, J=7.5 \mathrm{~Hz}, 4 \mathrm{H}, \mathrm{Ar}-\mathbf{H}), 7.17(\mathrm{t}, J=6.0 \mathrm{~Hz}, 6 \mathrm{H}, \mathrm{Ar}-\mathrm{H}), 6.69(\mathrm{~s}, 2 \mathrm{H}$, thiophene-H), $4.55(\mathrm{t}$, $J=5.5 \mathrm{~Hz}, 2 \mathrm{H}, \mathrm{OH}), 3.85\left(\mathrm{~d}, J=3.1 \mathrm{~Hz}, 4 \mathrm{H}, \mathrm{CH}_{2} \mathrm{NH}\right), 3.32\left(\mathrm{dd}, J=10.9,5.1 \mathrm{~Hz}, 2 \mathrm{H}, \mathrm{CH}_{2} \mathrm{OH}\right)$, $3.23\left(\mathrm{dd}, J=10.9,5.1 \mathrm{~Hz}, 2 \mathrm{H}, \mathrm{CH}_{2}-\mathrm{OH}\right), 2.75(\mathrm{q}, J=5.8 \mathrm{~Hz}, 2 \mathrm{H}, \mathrm{CHNH}), 2.68(J=13.4$, $\left.5.8 \mathrm{~Hz}, 2 \mathrm{H}, \mathrm{CH}_{2}-\mathrm{Ph}\right), 2.62\left(J=13.4,5.8 \mathrm{~Hz}, 2 \mathrm{H}, \mathrm{CH}_{2}-\mathrm{Ph}\right), 1.93(\mathrm{br}, 2 \mathrm{H}, \mathrm{NH}) ;{ }^{13} \mathrm{C}-\mathrm{NMR}$ $\left(126 \mathrm{MHz}, \mathrm{DMSO}-\mathrm{d}_{6}\right) \delta(\mathrm{ppm})={ }^{13} \mathrm{C}$ NMR $(126 \mathrm{MHz}, \mathrm{DMSO}-\mathrm{D} 6) \delta(\mathrm{ppm}) 143.60,139.69$, 129.32, 128.10, 125.79, 123.66, 62.20, 59.69, 45.77, 37.28; LCMS (ESI, m/z): found 410.20 $[\mathrm{M}+\mathrm{H}]^{+}$, exact mass 410.20 for $\mathrm{C}_{24} \mathrm{H}_{30} \mathrm{~N}_{2} \mathrm{O}_{2} \mathrm{~S}$; Anal. calcd. for $\mathrm{C}_{24} \mathrm{H}_{30} \mathrm{~N}_{2} \mathrm{O}_{2} \mathrm{~S}: \mathrm{C}, 70.21 ; \mathrm{H}$, 7.37; N, 6.82; found C, 70.15; $\mathrm{H}, 7.33 ; \mathrm{N}, 6.78$.

\subsection{2. (2S,2'S)-2,2'-((Thiophene-2,5-diylbis (methylene))bis(azanediyl))bis(2-phenylethan-1-ol) (L2)}

Following GP1, thiophene-2,5-dicarboaldehyde (3) and (S)-2-amino-2-phenylethan-1ol (4b) were reacted to produce chiral diamine alcohol (L2) as pale brown solid (256 $\mathrm{mg}$, $67 \%$ ); m.p. $100-103{ }^{\circ} \mathrm{C} ;[\alpha]_{D}^{25}=+87.13^{\circ}$ (c 0.124, MeOH); IR $\left(\mathrm{KBr}, \mathrm{cm}^{-1}\right)$ : 3292, 3061, 3030, 2918, 2863, 1602, 1492, 1453, 1337, 1025, $761 \mathrm{~cm}^{-1}$; ${ }^{1} \mathrm{H}-\mathrm{NMR}$ (500 MHz, DMSO- $d_{6}$ ): $\delta(\mathrm{ppm})=7.41-7.31(\mathrm{~m}, 6 \mathrm{H}, \mathrm{Ar}-\mathrm{H}), 7.28-7.21(\mathrm{~m}, 2 \mathrm{H}, \mathrm{Ar}-\mathrm{H}), 6.67(\mathrm{~s}, 2 \mathrm{H}$, thiophene-H), 4.88 $(\mathrm{s}, 2 \mathrm{H}, \mathrm{OH}), 3.72(\mathrm{dt}, J=8.4,4.3 \mathrm{~Hz}, 2 \mathrm{H}, \mathrm{CHNH}), 3.63\left(\mathrm{t}, J=7.7 \mathrm{~Hz}, 4 \mathrm{H}, \mathrm{CH}_{2} \mathrm{NH}\right), 3.58(\mathrm{~d}$, $\left.J=6.0 \mathrm{~Hz}, 2 \mathrm{H}, \mathrm{CH}_{2} \mathrm{~N}\right), 3.45\left(\mathrm{dd}, J=10.6,4.6 \mathrm{~Hz}, 2 \mathrm{H}, \mathrm{CH}_{2} \mathrm{OH}\right), 2.55(\mathrm{td}, J=7.3,3.7 \mathrm{~Hz}$, $2 \mathrm{H}, \mathrm{NH}) .{ }^{13} \mathrm{C}-\mathrm{NMR}\left(126 \mathrm{MHz}, \mathrm{DMSO}-d_{6}\right) \delta(\mathrm{ppm})=143.07,141.54,128.25,127.54,127.04$, 123.97, 66.44, 63.66, 45.67; LCMS (ESI, $\mathrm{m} / \mathrm{z}$ ): found $383.20[\mathrm{M}+\mathrm{H}]^{+}$, exact mass 382.17 for $\mathrm{C}_{22} \mathrm{H}_{26} \mathrm{~N}_{2} \mathrm{O}_{2} \mathrm{~S}$; Anal. calcd. for $\mathrm{C}_{22} \mathrm{H}_{26} \mathrm{~N}_{2} \mathrm{O}_{2} \mathrm{~S}: \mathrm{C}, 69.08 ; \mathrm{H}, 6.85 ; \mathrm{N}, 7.32$; found $\mathrm{C}, 69.13 ; \mathrm{H}$, $6.81 ; \mathrm{N}, 7.27$.

3.4.3. (2S, 2'S)-2,2'-((Thiophene-2,5-diylbis(methylene))bis(azanediyl))bis(3-methyl butan-1-ol) (L3)

Following GP1, thiophene-2,5-dicarboaldehyde (3) and (S)-2-amino-3-methylbutan-1$\mathrm{ol}(4 \mathrm{c})$ were reacted to produce chiral diamine alcohol (L3) as pale brown solid (204 mg, $65 \%) ;[\alpha]_{D}^{25}=-3.76^{\circ}$ (c $\left.0.158, \mathrm{MeOH}\right) ; \mathrm{IR}\left(\mathrm{KBr}, \mathrm{cm}^{-1}\right)$ : 3412, 3061, 3030, 2975, 2874, 1602, $1466,1336,1025,763 \mathrm{~cm}^{-1} ; 1 \mathrm{H}-\mathrm{NMR}\left(500 \mathrm{MHz}, \mathrm{DMSO}-d_{6}\right): \delta(\mathrm{ppm})=6.72(\mathrm{~s}, 2 \mathrm{H}$, thiopheneH), $4.37(\mathrm{t}, J=5.3 \mathrm{~Hz}, 2 \mathrm{H}, \mathrm{OH}), 3.88\left(\mathrm{~d}, J=14.1 \mathrm{~Hz}, 2 \mathrm{H}, \mathrm{CH}_{2} \mathrm{NH}\right), 3.80(\mathrm{~d}, J=14.1 \mathrm{~Hz}$, $\left.2 \mathrm{H}, \mathrm{CH}_{2} \mathrm{NH}\right) 3.44\left(\mathrm{dd}, J=10.8,4.3 \mathrm{~Hz}, 2 \mathrm{H}, \mathrm{CH}_{2} \mathrm{OH}\right), 3.32-3.27\left(\mathrm{~m}, 2 \mathrm{H}, \mathrm{CH}\left(\mathrm{CH}_{3}\right)_{2}, 3.29\right.$ $\left(\mathrm{dd}, J=10.8,4.3 \mathrm{~Hz}, 2 \mathrm{H}, \mathrm{CH}_{2} \mathrm{OH}\right), 2.29(\mathrm{dt}, J=6.1,5.0 \mathrm{~Hz}, 2 \mathrm{H}, \mathrm{CH}-\mathrm{N}), 1.80(\mathrm{~s}, 2 \mathrm{H}, \mathrm{NH})$, $1.73(\mathrm{~m}, 2 \mathrm{H}, \mathrm{CH}), 0.85\left(\mathrm{dd}, J=10.2,6.9 \mathrm{~Hz}, 12 \mathrm{H}, \mathrm{CH}_{3}\right) .{ }^{13} \mathrm{C}-\mathrm{NMR}\left(126 \mathrm{MHz}, \mathrm{DMSO}-d_{6}\right)$ : $\delta(\mathrm{ppm})=144.02,123.39,63.06,60.09,46.42,28.25,18.77,18.62 ; \mathrm{LCMS}(\mathrm{ESI}, \mathrm{m} / \mathrm{z})$ : found $315.20[\mathrm{M}+\mathrm{H}]^{+}$, exact mass 314.20 for $\mathrm{C}_{16} \mathrm{H}_{30} \mathrm{~N}_{2} \mathrm{O}_{2} \mathrm{~S}$; Anal. calcd. for $\mathrm{C}_{16} \mathrm{H}_{30} \mathrm{~N}_{2} \mathrm{O}_{2} \mathrm{~S}: \mathrm{C}$, $61.11 ; \mathrm{H}, 9.62 ; \mathrm{N}, 8.91$; found $\mathrm{C}, 60.99 ; \mathrm{H}, 9.69 ; \mathrm{N}, 8.93$.

3.4.4. (2S,2'S)-2,2'-((Thiophene-2,5-diylbis(methylene))bis(azanediyl))bis(3,3-dimethyl butan-1-ol) (L4)

Following GP1, thiophene-2,5-dicarboaldehyde (3) and (S)-2-amino-3,3-dimethylbutan1-ol (4d) were reacted to produce chiral diamine alcohol (L4) as pale yellow solid (229mg, $67 \%$ ); m.p. $72-174{ }^{\circ} \mathrm{C} ;[\alpha]_{D}^{25}=-96.31^{\circ}$ (c $\left.0.072, \mathrm{MeOH}\right)$; IR $\left(\mathrm{KBr}, \mathrm{cm}^{-1}\right)$ : 3330, 3201, 3131, 3058, 2951, 2866, 1604, 1481, 1392, 1002, $742 \mathrm{~cm}^{-1}$; ${ }^{1} \mathrm{H}-\mathrm{NMR}$ (500 MHz, DMSO$\left.d_{6}\right): \delta(\mathrm{ppm})=6.72(\mathrm{~s}, 2 \mathrm{H}$, thiophene-H), $4.36(\mathrm{t}, J=5.2 \mathrm{~Hz}, 2 \mathrm{H}, \mathrm{OH}), 4.06(\mathrm{dd}, J=13.9$, 
$\left.5.1 \mathrm{~Hz}, 2 \mathrm{H}, \mathrm{CH}_{2} \mathrm{NH}\right), 3.77\left(\mathrm{dd}, J=13.9,8.3 \mathrm{~Hz}, 2 \mathrm{H}, \mathrm{CH}_{2} \mathrm{NH}\right) 3.63(\mathrm{dd}, J=11.3,4.4 \mathrm{~Hz}, 2 \mathrm{H}$, $\left.\mathrm{CH}_{2} \mathrm{OH}\right), 3.34\left(\mathrm{dd}, J=10.2,4.0 \mathrm{~Hz}, 2 \mathrm{H}, \mathrm{CH}_{2} \mathrm{OH}\right), 2.12(\mathrm{ddd}, J=7.2,5.9,4.0 \mathrm{~Hz}, 2 \mathrm{H}, \mathrm{CHNH}$ ), $1.73(\mathrm{td}, J=7.9,5.3 \mathrm{~Hz}, 2 \mathrm{H}, \mathrm{NH}), 0.87\left(\mathrm{~s}, 18 \mathrm{H}, \mathrm{CH}_{3}\right) ;{ }^{13} \mathrm{C}-\mathrm{NMR}\left(126 \mathrm{MHz}, \mathrm{DMSO}-d_{6}\right)$ : $\delta(\mathrm{ppm})=144.08,123.27,66.71,60.22,48.61,34.19,27.26 ; \mathrm{LCMS}(\mathrm{ESI}, \mathrm{m} / \mathrm{z})$ : found 343.25 $[\mathrm{M}+\mathrm{H}]^{+}$, exact mass 342.23 for $\mathrm{C}_{18} \mathrm{H}_{34} \mathrm{~N}_{2} \mathrm{O}_{2} \mathrm{~S}$; Anal. calcd. for $\mathrm{C}_{18} \mathrm{H}_{34} \mathrm{~N}_{2} \mathrm{O}_{2} \mathrm{~S}: \mathrm{C}, 63.12 ; \mathrm{H}$, $10.01 ; \mathrm{N}, 8.18$; found $\mathrm{C}, 62.98 ; \mathrm{H}, 10.11 ; \mathrm{N}, 8.15$.

3.4.5. (2S,2'S)-2,2' -((Thiophene-2,5-diylbis(methylene))bis(azanediyl) )bis(butan-1-ol) (L5)

Following GP1, thiophene-2,5-dicarboaldehyde (3) and (S)-2-aminobutan-1-ol (4e) were reacted to produce chiral diamine alcohol (L5) as a pale yellow solid (200 $\mathrm{mg}, 70 \%)$; m.p. 94-95 ${ }^{\circ} \mathrm{C} ;[\alpha]_{D}^{25}=+32.73^{\circ}$ (c 0.107, $\left.\mathrm{MeOH}\right)$; IR (KBr, cm $\left.{ }^{-1}\right): 3309,3089,3131,3058,2958$, 2855, 1604, 1458, 1428, 1021, $764 \mathrm{~cm}^{-1}$. ${ }^{1} \mathrm{H}-\mathrm{NMR}\left(500 \mathrm{MHz}, \mathrm{DMSO}-d_{6}\right): \delta(\mathrm{ppm})=6.75$ (s, 2H, thiophene-H), $4.44(\mathrm{br}, 2 \mathrm{H}, \mathrm{OH}), 3.85\left(\mathrm{~s}, 4 \mathrm{H}, \mathrm{CH}_{2} \mathrm{NH}\right), 3.39(\mathrm{dd}, J=10.7,5.0 \mathrm{~Hz}$, $\left.2 \mathrm{H}, \mathrm{CH}_{2} \mathrm{OH}\right), 3.29\left(\mathrm{dd}, J=10.7,5.8 \mathrm{~Hz}, 2 \mathrm{H}, \mathrm{CH}_{2} \mathrm{OH}\right), 2.45(\mathrm{p}, J=5.8 \mathrm{~Hz}, 2 \mathrm{H}, \mathrm{CHNH})$, 1.44-1.30 (m, 4H, $\left.\mathrm{CH}_{2} \mathrm{CH}_{3}\right), 0.84\left(\mathrm{t}, J=7.5 \mathrm{~Hz}, 6 \mathrm{H}, \mathrm{CH}_{3}\right) ;{ }^{13} \mathrm{C}-\mathrm{NMR}\left(126 \mathrm{MHz}, \mathrm{DMSO}-d_{6}\right)$ $\delta(\mathrm{ppm})=143.67,123.65,62.17,59.06,45.47,23.41,10.00 ; \mathrm{LCMS}(\mathrm{ESI}, \mathrm{m} / \mathrm{z})$ : found 287.20 $[\mathrm{M}+\mathrm{H}]^{+}$, exact mass 286.17 for $\mathrm{C}_{14} \mathrm{H}_{26} \mathrm{~N}_{2} \mathrm{O}_{2} \mathrm{~S}$; Anal. calcd. for $\mathrm{C}_{14} \mathrm{H}_{26} \mathrm{~N}_{2} \mathrm{O}_{2} \mathrm{~S}$ : C, 58.71; $\mathrm{H}$, $9.15 ; \mathrm{N}, 9.78$; found $\mathrm{C}, 58.61 ; \mathrm{H}, 9.29 ; \mathrm{N}, 9.71$.

\subsection{General Procedures for the Synthesis of Racemic Nitroaldol Products (Rac $8 a-m$ )}

General Procedure (GP2): To a solution of aldehyde 6a-m (1.0 equiv.) and nitromethane 7 (1.4 equiv.) in ethanol ( $3 \mathrm{~mL}$ ) sodiumacetate trihydrate (0.6 equiv.) was added at room temperature as per the literature [61]. The resulting suspension was stirred for $72 \mathrm{~h}$ and then filtered. The filtrate was concentrated in vacuo and the residue was purified by flash column chromatography using 10-15\% ethylacetate/ $n$-hexane as eluent to afford racemic products rac8a-m, in excellent yields ( $\geq 0 \%)$.

\subsection{General Procedure for the Catalytic Asymmetric Henry Reaction (8a-m)}

General Procedure (GP3): A small $8 \mathrm{~mL}$ vial under nitrogen atmosphere was charged with ligand 4 (14mg, 0.041 mmol, $20 \mathrm{~mol} \%), \mathrm{Cu}(\mathrm{OAc})_{2} \cdot \mathrm{H}_{2} \mathrm{O}(8 \mathrm{mg}, 0.04 \mathrm{mmol}, 20 \mathrm{~mol} \%)$ and ethanol $(2 \mathrm{~mL})$. The solution was stirred for $2 \mathrm{~h}$ at room temperature to obtain a blue solution of $\mathbf{L} 4-\mathrm{Cu}(\mathrm{OAc})_{2} \cdot \mathrm{H}_{2} \mathrm{O}$ complex. The aldehyde $6 \mathbf{a}-\mathbf{m}(0.2 \mathrm{mmol})$ were then added to this blue colored solution of $\mathrm{L} 4-\mathrm{Cu}(\mathrm{OAc})_{2} \cdot \mathrm{H}_{2} \mathrm{O}$ complex and stirred for 20 min at room temperature followed by addition of nitromethane $7(122 \mathrm{mg}, 2 \mathrm{mmol})$ and the reaction mixture was left stirring for the $24-48 \mathrm{~h}$. The solvent was then removed under reduced pressure and the residue was directly purified on 100 mesh silica gel column eluting by $10-15 \%$ EtOAc/petroleum ether to obtain the corresponding product chiral nitroaldol product $8 \mathbf{a}-\mathbf{m}$.

\subsection{1. (R)-(+)-2-Nitro-1-(2-nitrophenyl)ethan-1-ol (8a)}

2-Nitrobenzaldehyde $6 \mathbf{a}(30.22 \mathrm{mg}, 0.2 \mathrm{mmol})$ and nitromethane 7 (122 $\mathrm{mg}, 2 \mathrm{mmol})$ were reacted according to the GP3 to yield product $8 \mathbf{a}$ as yellow oil, isolated yield (42 mg, 99\%). Enantiomeric excess (ee) was determined by chiral HPLC [Chiracel OD-H column), 90.0\% n-hexane $/ i$-PrOH, $0.8 \mathrm{~mL} / \mathrm{min}$; $\mathrm{t}_{\text {major }}=18.19 \mathrm{~min} . ; \mathrm{t}_{\operatorname{minor}}=20.56 \mathrm{~min}$; $\lambda=254 \mathrm{~nm}$; $94.56 \%$ ee; $[\alpha]_{\mathrm{D}}^{20}=+239.3^{\circ}$ (c 1.0, $\mathrm{CH}_{2} \mathrm{Cl}_{2}$ ); Ref. [62] $[\alpha]_{\mathrm{D}}^{20}=+237.0^{\circ}$ (c 1.0, $\mathrm{CH}_{2} \mathrm{Cl}_{2}$ ); ${ }^{1} \mathrm{H}-\mathrm{NMR}\left(500 \mathrm{MHz}, \mathrm{CDCl}_{3}\right): \delta(\mathrm{ppm})=8.07(\mathrm{~d}, J=8.2 \mathrm{~Hz}, 1 \mathrm{H}, \mathrm{Ar}-\mathrm{H}), 7.95(\mathrm{~d}, J=7.9 \mathrm{~Hz}, 1 \mathrm{H}$, Ar-H), 7.77-7.72 (m, 1H, Ar-H), 7.58-7.52 (m, 1H, Ar-H), 6.04 (d, J=9.2 Hz, 1H, CHOH), $4.86\left(\mathrm{dd}, 1 \mathrm{H}, J=13.89,2.43 \mathrm{~Hz}, \mathrm{CH}_{2} \mathrm{NO}_{2}\right), 4.55\left(\mathrm{dd}, 1 \mathrm{H}, J=13.75,9.02 \mathrm{~Hz}, \mathrm{CH}_{2} \mathrm{NO}_{2}\right), 3.28$ $(\mathrm{s}, 1 \mathrm{H}, \mathrm{OH}) ;{ }^{13} \mathrm{C}-\mathrm{NMR}\left(125 \mathrm{MHz} \mathrm{CDCl}_{3}\right): \delta(\mathrm{ppm})=147.28,134.54,134.13,129.83,128.83$, $125.15,80.17,66.91$; all the analytical data are in accordance with the reported literature [62].

\subsection{2. (R)-(-)-2-Nitro-1-(4-nitrophenyl)ethan-1-ol (8b)}

4-Nitrobenzaldehyde $6 \mathrm{~b}(30.22 \mathrm{mg}, 0.2 \mathrm{mmol})$ and nitromethane 7 (122 $\mathrm{mg}, 2 \mathrm{mmol})$ were reacted according to the GP3 to yield product $\mathbf{8 b}$ as yellow oil, isolated yield (40.7 $\mathrm{mg}$, 
96\%). Enantiomeric excess (ee) was determined by chiral HPLC [Chiracel OD-H column), 85.0\% $n$-hexane $/ i$-PrOH, $1.0 \mathrm{~mL} / \mathrm{min}$.; $\mathrm{t}_{\text {major }}=15.89 \mathrm{~min}$.; $\mathrm{t}_{\text {minor }}=20.19 \mathrm{~min}$; $\lambda=254 \mathrm{~nm}] ; 81.32 \%$ ee; $[\alpha]_{\mathrm{D}}^{20}=-18.7^{\circ}\left(\mathrm{c} 0.5, \mathrm{CH}_{2} \mathrm{Cl}_{2}\right)$; Ref. [40] $[\alpha]_{\mathrm{D}}^{20}=-39.1^{\circ}$ (c 0.98 , $\left.\mathrm{CH}_{2} \mathrm{Cl}_{2}\right) ; \delta(\mathrm{ppm})=8.24(\mathrm{~d}, J=8.2 \mathrm{~Hz}, 2 \mathrm{H}, \mathrm{Ar}-\mathrm{H}), 7.62(\mathrm{~d}, J=8.7 \mathrm{~Hz}, 2 \mathrm{H}, \mathrm{Ar}-\mathrm{H}), 5.60(\mathrm{dd}$, $J=8.5,3.9 \mathrm{~Hz}, 1 \mathrm{H}, \mathrm{CHOH}), 4.64-4.54\left(\mathrm{~m}, 2 \mathrm{H}, \mathrm{CH}_{2} \mathrm{NO}_{2}\right), 3.48-3.40(\mathrm{~m}, 1 \mathrm{H}, \mathrm{OH}) ;{ }^{13} \mathrm{C}-\mathrm{NMR}$ $\left(125 \mathrm{MHz}, \mathrm{CDCl}_{3}\right): \delta(\mathrm{ppm})=148.27,145.11,127.09,124.33,80.73,70.10$. All the analytical data are in accordance with the reported literature $[40,61]$.

\subsection{3. (R)-(-)-2-Nitro-1-(3-nitrophenyl)ethan-1-ol (8c)}

3-Nitrobenzaldehyde $6 \mathrm{c}(30.22 \mathrm{mg}, 0.2 \mathrm{mmol})$ and nitromethane 7 (122 mg, $2 \mathrm{mmol})$ were reacted according to the GP3 to yield product $8 \mathrm{c}$ as yellow oil, isolated yield $(38.61 \mathrm{mg}$, 91\%). Enantiomeric excess (ee) was determined by chiral HPLC [Chiracel OD-H column), $85.0 \%$-hexane $/ i-\mathrm{PrOH}, 1.0 \mathrm{~mL} / \mathrm{min}$; $\mathrm{t}_{\mathrm{major}}=15.51 \mathrm{~min}$.; $\mathrm{t}_{\mathrm{minor}}=17.73 \mathrm{~min}$; $\left.\lambda=254 \mathrm{~nm}\right]$; $81.20 \%$ ee; $[\alpha]_{\mathrm{D}}^{20}=-17.4^{\circ}$ (c $\left.0.5, \mathrm{CH}_{2} \mathrm{Cl}_{2}\right)$; Ref. [62] $[\alpha]_{\mathrm{D}}^{20}=-32.5^{\circ}$ (c $1.0, \mathrm{CH}_{2} \mathrm{Cl}_{2}$ ); ${ }^{1} \mathrm{H}-$ $\operatorname{NMR}\left(500 \mathrm{MHz}, \mathrm{CDCl}_{3}\right): \delta(\mathrm{ppm})=8.32(\mathrm{~s}, 1 \mathrm{H}, \mathrm{Ar}-\mathrm{H}), 8.21(\mathrm{~d}, J=8.2 \mathrm{~Hz}, 1 \mathrm{H}, \mathrm{Ar}-\mathbf{H})$, $7.77(\mathrm{~d}, J=9.5 \mathrm{~Hz}, 1 \mathrm{H}, \mathrm{Ar}-\mathrm{H}), 7.60(\mathrm{td}, J=7.9,2.0 \mathrm{~Hz}, 1 \mathrm{H}, \mathrm{Ar}-\mathrm{H}), 5.61(\mathrm{~d}, J=9.4 \mathrm{~Hz}, 1 \mathrm{H}$, $\mathrm{CHOH}), 4.66-4.56\left(\mathrm{~m}, 2 \mathrm{H}, \mathrm{CH}_{2} \mathrm{NO}_{2}\right), 3.43$ (brs, $\left.1 \mathrm{H}, \mathrm{OH}\right) ;{ }^{13} \mathrm{C}-\mathrm{NMR}\left(125 \mathrm{MHz}, \mathrm{CDCl}_{3}\right)$ : $\delta(\mathrm{ppm})=148.70,140.44,132.14,130.25,123.93,121.28,80.84,69.96$; all the analytical data are in accordance with reported literature [62].

\subsection{4. (R)-(-)-1-(4-Bromophenyl)-2-nitroethan-1-ol (8d)}

4-Bromobenzaldehyde $6 \mathrm{~d}(37 \mathrm{mg}, 0.2 \mathrm{mmol})$ and nitromethane 7 (122 mg, $2 \mathrm{mmol}$ ) were reacted according to the GP3 to yield product $\mathbf{8 d}$ as yellow oil, isolated yield ( $39.4 \mathrm{mg}$, $80 \%$ ). Enantiomeric excess (ee) was determined by chiral HPLC [Chiracel OD-H column), $85.0 \% n$-hexane $/ i-\mathrm{PrOH}, 0.8 \mathrm{~mL} / \mathrm{min}$.; $\mathrm{t}_{\text {major }}=13.95 \mathrm{~min}$.; $\mathrm{t}_{\mathrm{minor}}=18.25 \mathrm{~min}$.; $\left.\lambda=254 \mathrm{~nm}\right]$; $76.36 \%$ ee; $[\alpha]_{\mathrm{D}}^{20}=-8.8^{\circ}\left(\mathrm{c} 0.25, \mathrm{CH}_{2} \mathrm{Cl}_{2}\right)$; Ref. [40] $[\alpha]_{\mathrm{D}}^{20}=-48.0^{\circ}\left(\mathrm{c} 0.95, \mathrm{CH}_{2} \mathrm{Cl}_{2}\right) ;{ }^{1} \mathrm{H}-$ $\operatorname{NMR}\left(500 \mathrm{MHz}, \mathrm{CDCl}_{3}\right): \delta(\mathrm{ppm})=7.54(\mathrm{~d}, J=8.4 \mathrm{~Hz}, 2 \mathrm{H}, \mathrm{Ar}-\mathrm{H}), 7.30(\mathrm{~d}, J=8.2 \mathrm{~Hz}$, $2 \mathrm{H}, \mathrm{Ar}-\mathrm{H}), 5.44(\mathrm{dd}, J=9.5,3 \mathrm{~Hz}, 1 \mathrm{H}, \mathrm{CHOH}), 4.57\left(\mathrm{dd}, J=13.5,9.5 \mathrm{~Hz}, 1 \mathrm{H}, \mathrm{CH}_{2} \mathrm{NO}_{2}\right)$, $4.49\left(\mathrm{dd}, J=13.5,9.5 \mathrm{~Hz}, 1 \mathrm{H}, \mathrm{CH}_{2} \mathrm{NO}_{2}\right), 2.94(\mathrm{~s}, 1 \mathrm{H}, \mathrm{OH}) ;{ }^{13} \mathrm{C}-\mathrm{NMR}\left(125 \mathrm{MHz}, \mathrm{CDCl}_{3}\right)$ : $\delta(\mathrm{ppm})=137.17,132.34,127.76,123.12,81.04,70.48$. all the analytical data are in accordance with the reported literature $[40,61]$.

\subsection{5. (R)-(-)-1-(Naphthalen-2-yl)-2-nitroethan-1-ol (8e)}

2-Naphthaldehyde 6 e $(31.24 \mathrm{mg}, 0.2 \mathrm{mmol})$ and nitromethane $7(122 \mathrm{mg}, 2 \mathrm{mmol})$ were reacted according to the GP3 to yield product $8 \mathrm{e}$ as yellow oil, isolated yield $(28.67 \mathrm{mg}$, $66 \%$ ). Enantiomeric excess (ee) was determined by chiral HPLC [Chiracel OD-H column), $80.0 \%$ n-hexane $/ i-\mathrm{PrOH}, 1.0 \mathrm{~mL} / \mathrm{min}$.; $\mathrm{t}_{\text {major }}=21.35 \mathrm{~min}$; $\mathrm{t}_{\mathrm{minor}}=30.55 \mathrm{~min}$; $\lambda=254 \mathrm{~nm}$; $75.12 \%$ ee; $[\alpha]_{\mathrm{D}}^{20}=-21.6^{\circ}$ (c $0.5, \mathrm{CH}_{2} \mathrm{Cl}_{2}$ ); Ref. $[62][\alpha]_{\mathrm{D}}^{20}=-51.5^{\circ}$ (c $\left.1.11, \mathrm{CH}_{2} \mathrm{Cl}_{2}\right) ;{ }^{1} \mathrm{H}-$ $\operatorname{NMR}\left(500 \mathrm{MHz}, \mathrm{CDCl}_{3}\right): \delta(\mathrm{ppm})=7.65-7.57(\mathrm{~m}, 4 \mathrm{H}, \mathrm{Ar}-\mathrm{H}), 7.31-7.17(\mathrm{~m}, 3 \mathrm{H}, \mathrm{Ar}-\mathbf{H})$, $5.37(\mathrm{~d}, J=9.6 \mathrm{~Hz}, 1 \mathrm{H}, \mathrm{CHOH}), 4.43\left(\mathrm{dd}, J=13.5,9.6 \mathrm{~Hz}, 1 \mathrm{H}, \mathrm{CH}_{2} \mathrm{NO}_{2}\right), 4.34(\mathrm{dd}, J=13.5$, $\left.9.6 \mathrm{~Hz}, 1 \mathrm{H}, \mathrm{CH}_{2} \mathrm{NO}_{2}\right), 2.76(\mathrm{~s}, 1 \mathrm{H}, \mathrm{OH}) ;{ }^{13} \mathrm{C}-\mathrm{NMR}\left(125 \mathrm{MHz}, \mathrm{CDCl}_{3}\right): \delta(\mathrm{ppm})=135.54$, $133.56,133.32,129.17,128.20,127.94,126.87,126.83,125.48,123.34,81.34,71.29$. All the analytical data are in accordance with the reported literature $[40,62]$.

3.6.6. (R)-(-)-2-Nitro-1-(4-(trifluoromethyl)phenyl)ethan-1-ol (8f)

4-(Trifluoromethyl) benzaldehyde $6 \mathbf{f}(34.82 \mathrm{mg}, 0.2 \mathrm{mmol})$ and nitromethane 7 (122 mg, $2 \mathrm{mmol}$ ) were reacted according to the GP3 to yield product $8 \mathrm{f}$ as yellow oil, isolated yield (38.57 mg, 82\%). Enantiomeric excess (ee) was determined by chiral HPLC [Chiracel OD-H column), $85.0 \% n$-hexane $/ i-\mathrm{PrOH}, 0.8 \mathrm{~mL} / \mathrm{min}$; $\mathrm{t}_{\text {major }}=9.26 \mathrm{~min}$; $\mathrm{t}_{\text {minor }}=11.62 \mathrm{~min}$; $\lambda=254 \mathrm{~nm}] ; 58.91 \%$ ee; $[\alpha]_{\mathrm{D}}^{20}=-32.7^{\circ}\left(\right.$ c $\left.0.5, \mathrm{CH}_{2} \mathrm{Cl}_{2}\right)$; Ref. [62] $[\alpha]_{\mathrm{D}}^{20}=-24.5^{\circ}$ (c 1.0, $\left.\mathrm{CH}_{2} \mathrm{Cl}_{2}\right) ;{ }^{1} \mathrm{H}-\mathrm{NMR}\left(500 \mathrm{MHz}, \mathrm{CDCl}_{3}\right): \delta(\mathrm{ppm})=7.68(\mathrm{~d}, J=8.4 \mathrm{~Hz}, 2 \mathrm{H}, \mathrm{Ar}-\mathrm{H}), 7.55(\mathrm{~d}$, $J=8.0 \mathrm{~Hz}, 2 \mathrm{H}, \mathrm{Ar}-\mathrm{H}), 5.55(\mathrm{~d}, J=9.2 \mathrm{~Hz}, 1 \mathrm{H}, \mathrm{CHOH}), 4.62-4.52\left(\mathrm{~m}, 2 \mathrm{H}, \mathrm{CH}_{2} \mathrm{NO}_{2}\right), 3.06(\mathrm{~s}$, 
$1 \mathrm{H}, \mathrm{OH}) ;{ }^{13} \mathrm{C}-\mathrm{NMR}\left(125 \mathrm{MHz}, \mathrm{CDCl}_{3}\right): \delta(\mathrm{ppm})=142.03,126.50,126.17,126.15,81.00,70.44$. All the analytical data are in accordance with the reported literature [61,62].

\subsection{7. (R)-(-)-1-(2,4-Dichlorophenyl)-2-nitroethan-1-ol (8g)}

2,4-Dichlorobenzaldehyde $6 \mathrm{~g}$ ( $35 \mathrm{mg}, 0.2 \mathrm{mmol})$ and nitromethane $7(122 \mathrm{mg}, 2 \mathrm{mmol})$ were reacted according to the GP3 to yield product $8 \mathrm{~g}$ as yellow oil, isolated yield ( $40.60 \mathrm{mg}$, $86 \%$ ). Enantiomeric excess (ee) was determined by chiral HPLC [Chiracel OD-H column), $95.0 \%$-hexane $/ i-\mathrm{PrOH}, 0.8 \mathrm{~mL} / \mathrm{min}$.; $\mathrm{t}_{\text {major }}=18.45 \mathrm{~min}$.; $\mathrm{t}_{\text {minor }}=19.31 \mathrm{~min}$.; $\lambda=254 \mathrm{~nm}$; $53.02 \%$ ee; $[\alpha]_{\mathrm{D}}^{20}=-13.7^{\circ}\left(\mathrm{c} 0.5, \mathrm{CH}_{2} \mathrm{Cl}_{2}\right)$; Ref. [62] $[\alpha]_{\mathrm{D}}^{20}=-50.7^{\circ}\left(\mathrm{c} 1.0, \mathrm{CH}_{2} \mathrm{Cl}_{2}\right) ;{ }^{1} \mathrm{H}-\mathrm{NMR}$ $\left(500 \mathrm{MHz}, \mathrm{CDCl}_{3}\right): \delta(\mathrm{ppm})=7.62(\mathrm{~d}, J=8.4 \mathrm{~Hz}, 1 \mathrm{H}, \mathrm{Ar}-\mathrm{H}), 7.41(\mathrm{~d}, J=2.1 \mathrm{~Hz}, \mathrm{H}, \mathrm{Ar}-\mathrm{H})$, $7.34(\mathrm{dd}, J=8.4,2.1 \mathrm{~Hz}, 1 \mathrm{H}, \mathrm{Ar}-\mathrm{H}) 5.80(\mathrm{~d}, J=9.5 \mathrm{~Hz}, 1 \mathrm{H}, \mathrm{CHOH}), 4.65(\mathrm{dd}, J=13.7$, $\left.2.4 \mathrm{~Hz}, 1 \mathrm{H}, \mathrm{CH}_{2} \mathrm{NO}_{2}\right), 4.42\left(\mathrm{dd}, J=13.7,9.5 \mathrm{~Hz}, 1 \mathrm{H}, \mathrm{CH}_{2} \mathrm{NO}_{2}\right) 3.08(\mathrm{~d}, J=4.3 \mathrm{~Hz}, 1 \mathrm{H}, \mathrm{OH})$; ${ }^{13} \mathrm{C}-\mathrm{NMR}\left(125 \mathrm{MHz}, \mathrm{CDCl}_{3}\right): \delta(\mathrm{ppm})=135.40,134.24,132.22,129.65,128.73,128.13,79.18$, 67.56. All the analytical data are in accordance with the reported literature $[61,62]$.

\subsection{8. (R)-(-)-1-(4-Chlorophenyl)-2-nitroethan-1-ol (8h)}

4-Chlorobenzaldehyde $6 \mathrm{~h}(28 \mathrm{mg}, 0.2 \mathrm{mmol})$ and nitromethane 7 (122 mg, $2 \mathrm{mmol})$ were reacted according to the GP3 to yield product $8 \mathrm{~h}$ as yellow oil, isolated yield $(26.61 \mathrm{mg}$, $66 \%$ ). Enantiomeric excess (ee) was determined by chiral HPLC [Chiracel OD-H column), $90.0 \%$-hexane $/ i-\mathrm{PrOH}, 0.8 \mathrm{~mL} / \mathrm{min}$.; $\mathrm{t}_{\text {major }}=17.49 \mathrm{~min}$.; $\mathrm{t}_{\mathrm{minor}}=22.25 \mathrm{~min}$.; $\left.\lambda=254 \mathrm{~nm}\right]$; $73.05 \%$ ee; $[\alpha]_{\mathrm{D}}^{20}=-11.6^{\circ}$ (c $\left.0.5, \mathrm{CH}_{2} \mathrm{Cl}_{2}\right)$; Ref. [61] $[\alpha]_{\mathrm{D}}^{20}=-34.7^{\mathrm{o}}\left(\right.$ c $\left.1.0, \mathrm{CH}_{2} \mathrm{Cl}_{2}\right)$; ${ }^{1} \mathrm{H}-$ $\operatorname{NMR}\left(500 \mathrm{MHz}, \mathrm{CDCl}_{3}\right): \delta(\mathrm{ppm})=7.37(\mathrm{~d}, J=8.7 \mathrm{~Hz}, 2 \mathrm{H}, \mathrm{Ar}-\mathbf{H}), 7.41(\mathrm{~d}, J=7.0 \mathrm{~Hz}$, $2 \mathrm{H}, \mathrm{Ar}-\mathrm{H}), 5.44(\mathrm{~d}, J=9.6 \mathrm{~Hz}, 1 \mathrm{H}, \mathrm{CHOH}), 4.57\left(\mathrm{ddd}, J=13.3,9.5,1.1 \mathrm{~Hz}, 1 \mathrm{H}, \mathrm{CH}_{2} \mathrm{NO}_{2}\right)$, 4.49 (ddd, $\left.J=13.3,3.0,1.1 \mathrm{~Hz}, 1 \mathrm{H}, \mathrm{CH}_{2} \mathrm{NO}_{2}\right), 3.06(\mathrm{~s}, 1 \mathrm{H}, \mathrm{OH}) ;{ }^{13} \mathrm{C}-\mathrm{NMR}(125 \mathrm{MHz}$, $\left.\mathrm{CDCl}_{3}\right): \delta(\mathrm{ppm})=136.67,134.95,129.35,127.46,81.11,70.42$. All the analytical data are in accordance with the reported literature $[40,61]$.

\subsection{9. (R)-(-)-1-(4-Fluorophenyl)-2-nitroethan-1-ol (8i)}

4-Fluorbenzaldehyde $6 \mathbf{i}(24.82 \mathrm{mg}, 0.2 \mathrm{mmol})$ and nitromethane 7 (122 mg, $2 \mathrm{mmol})$ were reacted according to the GP3 to yield product $8 \mathbf{i}$ as yellow oil, isolated yield ( $27.77 \mathrm{mg}$, $75 \%$ ). Enantiomeric excess (ee) was determined by chiral HPLC [Chiracel OD-H column), $85.0 \%$-hexane $/ i-\mathrm{PrOH}, 0.5 \mathrm{~mL} / \mathrm{min}$.; $\mathrm{t}_{\text {major }}=16.20 \mathrm{~min}$.; $\mathrm{t}_{\mathrm{minor}}=18.89 \mathrm{~min}$; $\lambda=254 \mathrm{~nm}$; $60.89 \%$ ee; $[\alpha]_{\mathrm{D}}^{20}=-19.1^{\mathrm{o}}$ (c $0.5, \mathrm{CH}_{2} \mathrm{Cl}_{2}$ ); Ref. [62] $[\alpha]_{\mathrm{D}}^{20}=-25.7^{\mathrm{o}}\left(\right.$ c $\left.1.0, \mathrm{CH}_{2} \mathrm{Cl}_{2}\right) ;{ }^{1} \mathrm{H}-$ $\operatorname{NMR}\left(500 \mathrm{MHz}, \mathrm{CDCl}_{3}\right): \delta(\mathrm{ppm})=7.44-7.36(\mathrm{~m}, 2 \mathrm{H}, \mathrm{Ar}-\mathrm{H}), 7.10(\mathrm{~m}, 2 \mathrm{H}, \mathrm{Ar}-\mathrm{H}), 5.46(\mathrm{~d}$, $J=9.6 \mathrm{~Hz}, 1 \mathrm{H}, \mathrm{CHOH}), 4.59\left(\mathrm{dd}, J=13.4,9.5 \mathrm{~Hz}, 1 \mathrm{H}, \mathrm{CH}_{2} \mathrm{NO}_{2}\right), 4.50(\mathrm{dd}, J=13.4,9.5 \mathrm{~Hz}$, $\left.1 \mathrm{H}, \mathrm{CH}_{2} \mathrm{NO}_{2}\right), 2.89(\mathrm{~s}, 1 \mathrm{H}, \mathrm{OH}) . ;{ }^{13} \mathrm{C}-\mathrm{NMR}\left(125 \mathrm{MHz}, \mathrm{CDCl}_{3}\right): \delta(\mathrm{ppm})=164.05,162.08$, $133.99,127.96,127.89,116.28,116.10,81.25,70.47$. All the analytical data are in accordance with the reported literature [49,62].

\subsubsection{0. (R)-(-)-1-(4-Methoxyphenyl)-2-nitroethan-1-ol (8j)}

4-Methoxybenzaldehyde $6 \mathbf{j}(27.3 \mathrm{mg}, 0.2 \mathrm{mmol})$ and nitromethane 7 (122 mg, $2 \mathrm{mmol})$ were reacted according to the $\mathbf{G P} \mathbf{3}$ to yield product $8 \mathbf{j}$ as yellow oil, isolated yield ( $30.37 \mathrm{mg}$, $77 \%$ ). Enantiomeric excess (ee) was determined by chiral HPLC [Chiracel OD-H column), $90.0 \%$-hexane $/ i-\mathrm{PrOH}, 1.0 \mathrm{~mL} / \mathrm{min}$.; $\mathrm{t}_{\text {major }}=20.51 \mathrm{~min}$.; $\mathrm{t}_{\mathrm{minor}}=26.77 \mathrm{~min}$.; $\left.\lambda=254 \mathrm{~nm}\right]$; $63.89 \%$ ee; $[\alpha]_{\mathrm{D}}^{20}=-9.8^{\circ}$ (c 0.5, $\left.\mathrm{CH}_{3} \mathrm{OH}\right)$; Ref. [61] $[\alpha]_{\mathrm{D}}^{20}=-33.3^{\circ}\left(\mathrm{c} 1.0, \mathrm{CH}_{2} \mathrm{Cl}_{2}\right) ;{ }^{1} \mathrm{H}-\mathrm{NMR}$ $\left(500 \mathrm{MHz}, \mathrm{CDCl}_{3}\right): \delta(\mathrm{ppm})=7.33(\mathrm{~d}, J=8.6 \mathrm{~Hz}, 2 \mathrm{H}, \mathrm{Ar}-\mathrm{H}), 6.93(\mathrm{~d}, J=8.7 \mathrm{~Hz}, 2 \mathrm{H}, \mathrm{Ar}-\mathbf{H})$, $5.42(\mathrm{~d}, J=9.7 \mathrm{~Hz}, 1 \mathrm{H}, \mathrm{CHOH}), 4.61\left(\mathrm{dd}, J=13.2,9.7 \mathrm{~Hz}, 1 \mathrm{H} \mathrm{CH}_{2} \mathrm{NO}_{2}\right), 4.48(\mathrm{dd}, J=13.2$, $\left.9.7 \mathrm{~Hz}, 1 \mathrm{H}, \mathrm{CH}_{2} \mathrm{NO}_{2}\right), 3.81\left(\mathrm{~s}, 3 \mathrm{H}, \mathrm{OCH}_{3}\right) 2.74(\mathrm{~s}, 1 \mathrm{H}, \mathrm{OH}) ;{ }^{13} \mathrm{C}-\mathrm{NMR}\left(125 \mathrm{MHz}, \mathrm{CDCl}_{3}\right)$ : $\delta(\mathrm{ppm})=160.21,130.29,127.43,114.55,81.39,70.82,55.50$. All the analytical data are in accordance with the reported literature $[40,61]$.

\subsubsection{1. (R)-(-)-2-Nitro-1-(p-tolyl)ethan-1-ol (8k)}

4-Methybenzaldehyde 6k (24 mg, $0.2 \mathrm{mmol}$ ) and nitromethane 7 (122 mg, $2 \mathrm{mmol}$ ) were reacted according to the GP3 to yield product $8 \mathbf{k}$ as yellow oil, isolated yield ( $30.80 \mathrm{mg}$, 
85\%). Enantiomeric excess (ee) was determined by chiral HPLC [Chiracel OD-H column), $85.0 \%$ n-hexane $/ i-\mathrm{PrOH}, 0.5 \mathrm{~mL} / \mathrm{min}$.; $\mathrm{t}_{\text {major }}=20.96 \mathrm{~min}$.; $\mathrm{t}_{\mathrm{mino}} \mathrm{r}=26.99 \mathrm{~min}$.; $\lambda=254 \mathrm{~nm}$ ]; $81.29 \%$ ee; $[\alpha]_{\mathrm{D}}^{20}=-25.9^{\circ}\left(\right.$ c $\left.0.5, \mathrm{CH}_{2} \mathrm{Cl}_{2}\right)$; Ref. [62] $[\alpha]_{\mathrm{D}}^{20}=-31.5^{\circ}$ (c 1.0, $\left.\mathrm{CH}_{2} \mathrm{Cl}_{2}\right) ;{ }^{1} \mathrm{H}$ $\operatorname{NMR}\left(500 \mathrm{MHz}, \mathrm{CDCl}_{3}\right): \delta(\mathrm{ppm})=7.29(\mathrm{~d}, J=8.2 \mathrm{~Hz}, 2 \mathrm{H}, \mathrm{Ar}-\mathrm{H}), 7.21(\mathrm{~d}, J=8.2 \mathrm{~Hz}, 2 \mathrm{H}$, Ar-H), 5.43 (d, $J=9.8 \mathrm{~Hz}, 1 \mathrm{H}, \mathrm{CHOH}), 4.60\left(\mathrm{dd}, J=13.3,9.6 \mathrm{~Hz}, 1 \mathrm{H}, \mathrm{CH}_{2} \mathrm{NO}_{2}\right), 4.49$ (dd, $\left.J=13.3,9.6 \mathrm{~Hz}, 1 \mathrm{H}, \mathrm{CH}_{2} \mathrm{NO}_{2}\right), 2.79(\mathrm{~s}, 1 \mathrm{H}, \mathrm{OH}), 2.36\left(\mathrm{~s}, 1 \mathrm{H}, \mathrm{CH}_{3}\right) ;{ }^{13} \mathrm{C}-\mathrm{NMR}(125 \mathrm{MHz}$, $\left.\mathrm{CDCl}_{3}\right): \delta(\mathrm{ppm})=139.08,135.30,129.84,126.01,81.39,71.04,21.30$. All the analytical data are in accordance with the reported literature [61,62].

\subsubsection{2. (R)-(-)-2-Nitro-1-(m-tolyl)ethan-1-ol (81)}

3-Methybenzaldehyde 61 (24 mg, $0.2 \mathrm{mmol})$ and nitromethane 7 (122 mg, $2 \mathrm{mmol})$ were reacted according to the GP3 to yield product $\mathbf{8 1}$ as yellow oil, isolated yield ( $31.89 \mathrm{mg}$, $88 \%$ ). Enantiomeric excess (ee) was determined by chiral HPLC [Chiracel OD-H column), 90.0\% n-hexane $/ i$-PrOH, $0.5 \mathrm{~mL} / \mathrm{min}$; $\mathrm{t}_{\text {major }}=24.72 \mathrm{~min} . ; \mathrm{t}_{\operatorname{minor}}=29.21 \mathrm{~min}$; $\lambda=254 \mathrm{~nm}$; $60.81 \%$ ee; $[\alpha]_{\mathrm{D}}^{20}=-36.9^{\circ}$ (c $0.5, \mathrm{CH}_{2} \mathrm{Cl}_{2}$ ); Ref. [62] $[\alpha]_{\mathrm{D}}^{20}=-92.3^{\circ}$ (c 1.0, $\mathrm{CH}_{2} \mathrm{Cl}_{2}$ ); ${ }^{1} \mathrm{H}-\mathrm{NMR}$ $\left(500 \mathrm{MHz}, \mathrm{CDCl}_{3}\right): \delta(\mathrm{ppm})=\delta(\mathrm{ppm}) 7.32(\mathrm{q}, J=7.5 \mathrm{~Hz}, 2 \mathrm{H}, \mathrm{Ar}-\mathbf{H}), 7.26(\mathrm{~s}, 1 \mathrm{H}, \mathrm{Ar}-\mathbf{H})$ $7.24(\mathrm{~d}, J=7.7 \mathrm{~Hz}, 2 \mathrm{H}, \mathrm{Ar}-\mathrm{H}), 5.47(\mathrm{~d}, J=9.7 \mathrm{~Hz}, 1 \mathrm{H}, \mathrm{CHOH}), 4.64(\mathrm{dd}, J=13.3,9.6 \mathrm{~Hz}$, $\left.1 \mathrm{H}, \mathrm{CH}_{2} \mathrm{NO}_{2}\right), 4.54\left(\mathrm{dd}, J=13.3,9.6 \mathrm{~Hz}, 1 \mathrm{H}, \mathrm{CH}_{2} \mathrm{NO}_{2}\right), 2.88$ (s, 1H, OH), $2.41\left(\mathrm{~s}, 1 \mathrm{H}, \mathrm{CH}_{3}\right)$; ${ }^{13} \mathrm{C}-\mathrm{NMR}\left(125 \mathrm{MHz} \mathrm{CDCl}_{3}\right): \delta(\mathrm{ppm})=139.03,138.20,129.86,129.07,126.72,123.11,81.40$, $71.18,21.54$. All the analytical data are in accordance with the reported literature $[61,62]$.

\subsubsection{3. (R)-(-)-2-Nitro-1-phenylethan-1-ol (8m)}

Benzaldehyde $6 \mathrm{~m}(21.22 \mathrm{mg}, 0.2 \mathrm{mmol})$ and nitromethane 7 (122 mg, $2 \mathrm{mmol})$ were reacted according to the GP3 to yield product $8 \mathrm{~m}$ as yellow oil, isolated yield $(27.41 \mathrm{mg}$, $82 \%$ ). Enantiomeric excess (ee) was determined by chiral HPLC [Chiracel OD-H column), 90.0\% $n$-hexane $/ i$-PrOH, $0.8 \mathrm{~mL} / \mathrm{min}$.; $\mathrm{t}_{\text {major }}=17.94 \mathrm{~min}$.; $\mathrm{t}_{\text {minor }}=22.72 \mathrm{~min}$.; $\lambda=254 \mathrm{~nm}$; $89.22 \%$ ee; $[\alpha]_{\mathrm{D}}^{20}=-14.7^{\circ}$ (c $0.5, \mathrm{CH}_{2} \mathrm{Cl}_{2}$ ); Ref. [61] $[\alpha]_{\mathrm{D}}^{20}=-35.2^{\circ}$ (c 1.0, $\mathrm{CH}_{2} \mathrm{Cl}_{2}$ ); ${ }^{1} \mathrm{H}-\mathrm{NMR}$ $\left(500 \mathrm{MHz}, \mathrm{CDCl}_{3}\right): \delta(\mathrm{ppm})=\delta(\mathrm{ppm}) 7.44-7.34(\mathrm{~m}, 5 \mathrm{H}, \mathrm{Ar}-\mathrm{H}), 5.44(\mathrm{~d}, J=9.7 \mathrm{~Hz}, 1 \mathrm{H}$, $\mathrm{CHOH}), 4.57\left(\mathrm{dd}, J=13.7,3.5 \mathrm{~Hz}, 1 \mathrm{H}, \mathrm{CH}_{2} \mathrm{NO}_{2}\right), 4.49\left(\mathrm{dd}, J=13.7,3.5 \mathrm{~Hz}, 1 \mathrm{H}, \mathrm{CH}_{2} \mathrm{NO}_{2}\right)$, $3.08(\mathrm{~s}, 1 \mathrm{H}, \mathrm{OH}) ;{ }^{13} \mathrm{C}-\mathrm{NMR}\left(125 \mathrm{MHz}, \mathrm{CDCl}_{3}\right): \delta(\mathrm{ppm})=138.24,129.12,129.06,126.05$, $81.29,71.08$. All the analytical data are in accordance with the reported literature $[40,61]$.

\section{Conclusions}

In conclusion, a series of newly design and developed $C_{2}$-symmetric bis( $\beta$-amino alcohol) ligands (L1-L5) have been synthesized based on thiophene framework, and their asymmetric catalytic efficiency has been examined in asymmetric Henry reaction of nitromethane with a variety of substituted aromatic aldehydes successfully. $20 \mathrm{~mol} \%$ of L4: $\mathrm{Cu}(\mathrm{OAc})_{2} \cdot \mathrm{H}_{2} \mathrm{O}$ complex catalytic system was found to be the most efficient catalyst for asymmetric Henry reaction in ethanol at $25^{\circ} \mathrm{C}$. Our newly developed catalytic system is one of the robust processes which are capable of inducing chirality into nitroaldol condensation of nitromethane with several substituted aldehydes with moderate to excellent isolate yields (66-99\%) and enantioselectivity (53-95\% ee) at room temperature in ethanol as a green solvent. The easy catalyst synthesis, mild conditions, high enantioselectivity and chemical yield enhanced the potential application for this catalyst system. Our further investigations are currently ongoing for other chiral transformations using this catalytic system, and henceforth the outcome of this research will be communicated in the near future.

Supplementary Materials: The following are available online at https: / www.mdpi.com/article / 10.3390/catal11101208/s1, Figure S1: ${ }^{1} \mathrm{H}-\mathrm{NMR}$ and ${ }^{13} \mathrm{C}-\mathrm{NMR}$ for thiophene-2,5-diyldimethanol-2, Figure S2: ${ }^{1} \mathrm{H}-\mathrm{NMR}$ and ${ }^{13} \mathrm{C}-\mathrm{NMR}$ for thiophene-2,5-dicarbaldehyde-3, Figure S3: ${ }^{1} \mathrm{H}-\mathrm{NMR}$ and ${ }^{13} \mathrm{C}-\mathrm{NMR}$ for thiophene-2,5-bis-( $\beta$-amino alcohol) ligand-L1, Figure S4: ${ }^{1} \mathrm{H}-\mathrm{NMR}$ and ${ }^{13} \mathrm{C}-\mathrm{NMR}$ for thiophene-2,5-bis-( $\beta$-amino alcohol) ligand-L2, Figure S5: ${ }^{1} \mathrm{H}-\mathrm{NMR}$ and ${ }^{13} \mathrm{C}-\mathrm{NMR}$ for thiophene-2,5bis-( $\beta$-amino alcohol) ligand-L3, Figure S6: ${ }^{1} \mathrm{H}-\mathrm{NMR}$ and ${ }^{13} \mathrm{C}-\mathrm{NMR}$ for thiophene-2,5-bis-( $\beta$-amino 
alcohol) ligand-L4, Figure S7: ${ }^{1} \mathrm{H}-\mathrm{NMR}$ and ${ }^{13} \mathrm{C}-\mathrm{NMR}$ for thiophene-2,5-bis-( $\beta$-amino alcohol) ligandL5, Figure S8: ${ }^{1} \mathrm{H}-\mathrm{NMR}$ and ${ }^{13} \mathrm{C}-\mathrm{NMR}$ for Henry product-8a, Figure S9: ${ }^{1} \mathrm{H}-\mathrm{NMR}$ and ${ }^{13} \mathrm{C}-\mathrm{NMR}$ for Henry product-8b, Figure S10: ${ }^{1} \mathrm{H}-\mathrm{NMR}$ and ${ }^{13} \mathrm{C}-\mathrm{NMR}$ for Henry product-8c, Figure S11: ${ }^{1} \mathrm{H}-\mathrm{NMR}$ and ${ }^{13} \mathrm{C}$-NMR for Henry product-8d, Figure S12: ${ }^{1} \mathrm{H}-\mathrm{NMR}$ and ${ }^{13} \mathrm{C}-\mathrm{NMR}$ for Henry product-8e, Figure S13: ${ }^{1} \mathrm{H}-\mathrm{NMR}$ and ${ }^{13} \mathrm{C}-\mathrm{NMR}$ for Henry product-8f, Figure S14: ${ }^{1} \mathrm{H}-\mathrm{NMR}$ and ${ }^{13} \mathrm{C}-\mathrm{NMR}$ for Henry product-8g, Figure S15: ${ }^{1} \mathrm{H}-\mathrm{NMR}$ and ${ }^{13} \mathrm{C}-\mathrm{NMR}$ for Henry product-8h, Figure S16: ${ }^{1} \mathrm{H}-\mathrm{NMR}$ and ${ }^{13} \mathrm{C}-\mathrm{NMR}$ for Henry product-8i, Figure S17: ${ }^{1} \mathrm{H}-\mathrm{NMR}$ and ${ }^{13} \mathrm{C}-\mathrm{NMR}$ for Henry product-8j, Figure S18: ${ }^{1} \mathrm{H}-\mathrm{NMR}$ and ${ }^{13} \mathrm{C}-\mathrm{NMR}$ for Henry product-8k, Figure S19: ${ }^{1} \mathrm{H}-\mathrm{NMR}$ and ${ }^{13} \mathrm{C}-\mathrm{NMR}$ for Henry product-81, Figure S20: ${ }^{1} \mathrm{H}-\mathrm{NMR}$ and ${ }^{13} \mathrm{C}-\mathrm{NMR}$ for Henry product-8m, Figure S21: Chiral HPLC for Henry product-8a, Figure S22: Chiral HPLC for for Friedel Craft product-8b, Figure S23: Chiral HPLC for for Friedel Craft product-8c, Figure S24: Chiral HPLC for for Friedel Craft product8d, Figure S25: Chiral HPLC for for Friedel Craft product-8e, Figure S26: Chiral HPLC for for Friedel Craft product-8f, Figure S27: Chiral HPLC for for Friedel Craft product-8g, Figure S28: Chiral HPLC for for Friedel Craft product-8h, Figure S29: Chiral HPLC for for Friedel Craft product-8i, Figure S30: Chiral HPLC for for Friedel Craft product-8j, Figure S31: Chiral HPLC for for Friedel Craft product-8k, Figure S32: Chiral HPLC for for Friedel Craft product-81, Figure S33: Chiral HPLC for for Friedel Craft product-8m.

Author Contributions: Conceptualization, A.M.A.-M. and A.B.; Supervision, A.M.A.-M., A.B. and M.S.I.; methodology, A.S.A., M.S.I., M.A. and A.M.A.-M.; validation, M.S.I., A.S.A., S.A., M.A. and A.M.A.-M.; formal analysis, A.S.A.; M.S.I., M.A. and S.A.; investigation, A.S.A.; M.S.I., M.A. and S.A.; resources, A.M.A.-M. and A.B.; data cu-ration, A.S.A., M.S.I. and A.B.; writing-original draft preparation, M.S.I., A.B. and A.S.A.; writing-review and editing, M.S.I., A.B. and A.M.A.-M.; visualization, A.B., M.S.I., S.A. and M.A.; project administration, A.M.A.-M. and A.B.; funding acquisition, A.M.A.-M. and A.B.; Software A.S.A., M.S.I., M.A. and A.B.; All authors have read and agreed to the published version of the manuscript.

Funding: Researchers Supporting Project number (RSP-2021/64), King Saud University, Riyadh, Saudi Arabia.

Data Availability Statement: The data presented in this study are available in Supplementary Material.

Acknowledgments: The authors would like to extend their sincere appreciation to the Researchers Supporting Project (RSP-2021/64), King Saud University, Riyadh, Saudi Arabia.

Conflicts of Interest: The authors declare no conflict of interest.

\section{References}

1. Mikami, K.; Lautens, M. New Frontiers in Asymmetric Catalysis; John Wiley \& Sons: Hoboken, NJ, USA, 2007.

2. Jacobsen, E.N.; Pfaltz, A.; Yamamoto, H. Comprehensive Asymmetric Catalysis: Supplement 1; Springer Science \& Business Media: Berlin/Heidelberg, Germany, 2003; Volume 1.

3. Ojima, I. Catalytic Asymmetric Synthesis; John Wiley \& Sons: Hoboken, NJ, USA, 2010.

4. Christmann, M.; Bräse, S. Asymmetric Synthesis ii: More Methods and Applications; John Wiley \& Sons: Hoboken, NJ, USA, 2013.

5. Nag, A. Asymmetric Synthesis of Drugs and Natural Products; CRC Press: Boca Raton, FL, USA, 2018.

6. Blacker, J. Catalytic Asymmetric Synthesis, 2nd ed.; Ojima, I., Ed.; Wiley-VCH: New York, NY, USA, 2000; p. 864. ISBN 0-471-29805-0.

7. Babu, S.A. Exploitation of intramolecular glaser-eglinton-hay macrocyclization for the synthesis of new classes of optically active aza-oxo-thia polyether macrocycles from amino alcohol building blocks. Synlett 2017, 28, 253-259. [CrossRef]

8. Seebach, D.; Colvin, E.; Lehr, F.; Weller, T. Nitroaliphatic compounds-ideal intermediates in organic synthesis. Chimia 1979, 33, $1-18$.

9. Luzzio, F.A. The Henry reaction: Recent examples. Tetrahedron 2001, 57, 915-945. [CrossRef]

10. Henry, L. Nitro-alcohols. Compt. Rend. Hebd. Seances Acad. Sci. 1895, 120, 1265-1268.

11. Heaney, F. The nitro group in organic synthesis. Synthesis 2001, 2001, 2528. [CrossRef]

12. White, J.D.; Shaw, S. A new catalyst for the asymmetric Henry reaction: Synthesis of $\beta$-nitroethanols in high enantiomeric excess. Org. Lett. 2012, 14, 6270-6273. [CrossRef] [PubMed]

13. Sasai, H. The Henry (nitroaldol) reaction. In Comprehensive Organic Synthesis II, 2nd ed.; Knochel, P., Ed.; Elsevier: Amsterdam, The Netherlands, 2014; pp. 543-570.

14. Ginesta, X.; Pastó, M.; Pericas, M.A.; Riera, A. New stereodivergent approach to 3-amino-2, 3, 6-trideoxysugars. Enantioselective synthesis of daunosamine, ristosamine, acosamine, and epi-daunosamine. Org. Lett. 2003, 5, 3001-3004. [CrossRef]

15. Guo, Z.-L.; Deng, Y.-Q.; Zhong, S.; Lu, G. Enantioselective synthesis of (R)-salmeterol employing an asymmetric Henry reaction as the key step. Tetrahedron Asymmetry 2011, 22, 1395-1399. [CrossRef] 
16. Liu, L.; Zhang, S.; Xue, F.; Lou, G.; Zhang, H.; Ma, S.; Duan, W.; Wang, W. Catalytic enantioselective henry reactions of isatins: Application in the concise synthesis of (S)-(-)-spirobrassinin. Chem. Eur. J. 2011, 17, 7791-7795. [CrossRef]

17. Gogoi, N.; Boruwa, J.; Barua, N.C. A total synthesis of (-)-bestatin using Shibasaki's asymmetric Henry reaction. Tetrahedron Lett. 2005, 46, 7581-7582. [CrossRef]

18. Rao, D.S.; Shivani, K.; Padhi, S.K. Immobilized arabidopsis thaliana hydroxynitrile lyase-catalyzed retro-Henry reaction in the synthesis of (S)- $\beta$-nitroalcohols. Appl. Biochem. Biotechnol. 2021, 193, 560-576. [CrossRef]

19. Suami, T.; Sasai, H.; Matsuno, K.; Suzuki, N.; Fukuda, Y.; Sakanaka, O. Synthetic approach toward antibiotic tunicamycins-VI total synthesis of tunicamycins. Tetrahedron Lett. 1984, 25, 4533-4536. [CrossRef]

20. Sato, K.-I.; Akai, S.; Shoji, H.; Sugita, N.; Yoshida, S.; Nagai, Y.; Suzuki, K.; Nakamura, Y.; Kajihara, Y.; Funabashi, M. Stereoselective and efficient total synthesis of optically active tetrodotoxin from D-glucose. J. Org. Chem. 2008, 73, 1234-1242. [CrossRef]

21. Jakubec, P.; Cockfield, D.M.; Dixon, D.J. Total synthesis of (-)-nakadomarin A. J. Am. Chem. Soc. 2009, 131, 16632-16633. [CrossRef] [PubMed]

22. Jakubec, P.; Hawkins, A.; Felzmann, W.; Dixon, D.J. Total synthesis of manzamine A and related alkaloids. J. Am. Chem. Soc. 2012, 134, 17482-17485. [CrossRef] [PubMed]

23. Sasai, H.; Suzuki, T.; Arai, S.; Arai, T.; Shibasaki, M. Basic character of rare earth metal alkoxides. Utilization in catalytic carbon-carbon bond-forming reactions and catalytic asymmetric nitroaldol reactions. J. Am. Chem. Soc. 1992, 114, 4418-4420. [CrossRef]

24. Ananthi, N.; Velmathi, S. Asymmetric Henry reaction catalysed by transition metal complexes: A short review. ChemInform 2013, 44, 87-108.

25. Alvarez-Casao, Y.; Marques-Lopez, E.; Herrera, R.P. Organocatalytic enantioselective Henry reactions. Symmetry 2011, 3, $220-245$. [CrossRef]

26. Xiong, Y.; Wang, F.; Huang, X.; Wen, Y.; Feng, X. A new copper (I)—tetrahydrosalen-catalyzed asymmetric Henry reaction and its extension to the synthesis of (S)-Norphenylephrine. Chem. Eur. J. 2007, 13, 829-833. [CrossRef]

27. Arai, T.; Watanabe, M.; Yanagisawa, A. Practical Asymmetric Henry reaction catalyzed by a chiral diamine-Cu (OAc) 2 complex. Org. Lett. 2007, 9, 3595-3597. [CrossRef]

28. Evans, D.A.; Seidel, D.; Rueping, M.; Lam, H.W.; Shaw, J.T.; Downey, C.W. A new copper acetate-bis (oxazoline)-catalyzed, enantioselective Henry reaction. J. Am. Chem. Soc. 2003, 125, 12692-12693. [CrossRef] [PubMed]

29. Sasai, H.; Suzuki, T.; Itoh, N.; Shibasaki, M. Catalytic asymmetric nitroaldol reactions. A new practical method for the preparation of the optically active lanthanum complex. Tetrahedron Lett. 1993, 34, 851-854. [CrossRef]

30. Trost, B.M.; Yeh, V.S. A dinuclear Zn catalyst for the asymmetric nitroaldol (Henry) reaction. Angew. Chem. 2002, 114, 889-891. [CrossRef]

31. Li, H.; Wang, B.; Deng, L. Enantioselective nitroaldol reaction of $\alpha$-ketoesters catalyzed by Cinchona alkaloids. J. Am. Chem. Soc. 2006, 128, 732-733. [CrossRef] [PubMed]

32. Liu, X.-G.; Jiang, J.-J.; Shi, M. Development of axially chiral bis (arylthiourea)-based organocatalysts and their application in the enantioselective Henry reaction. Tetrahedron Asymmetry 2007, 18, 2773-2781. [CrossRef]

33. Otevrel, J.; Bobal, P. Biphenyl-based bis (thiourea) organocatalyst for asymmetric and syn-selective Henry reaction. Synthesis 2017, 49, 593-603.

34. Chinchilla, R.; Nájera, C.; Sánchez-Agulló, P. Enantiomerically pure guanidine-catalysed asymmetric nitroaldol reaction. Tetrahedron Asymmetry 1994, 5, 1393-1402. [CrossRef]

35. Tetour, D.; Novotná, M.; Hodačová, J. Enantioselective Henry reaction catalyzed by copper(II) complex of bis (trans-cyclohexane-1, 2-diamine)-based ligand. Catalysts 2021, 11, 41. [CrossRef]

36. Bandini, M.; Piccinelli, F.; Tommasi, S.; Umani-Ronchi, A.; Ventrici, C. Highly enantioselective nitroaldol reaction catalyzed by new chiral copper complexes. Chem. Commun. 2007, 6, 616-618. [CrossRef]

37. Tanaka, K.; Hachiken, S. Enantioselective Henry reaction catalyzed by trianglamine-Cu(OAc) ${ }_{2}$ complex under solvent-free conditions. Tetrahedron Lett. 2008, 49, 2533-2536. [CrossRef]

38. Kowalczyk, R.; Sidorowicz, Ł.; Skarżewski, J. Asymmetric Henry reaction catalyzed by chiral secondary diamine-copper(II) complexes. Tetrahedron Asymmetry 2008, 19, 2310-2315. [CrossRef]

39. Kowalczyk, R.; Skarżewski, J. Asymmetric nitroaldol reaction catalyzed by copper-diamine complexes: Selective construction of two contiguous stereogenic centers. Tetrahedron Asymmetry 2009, 20, 2467-2473. [CrossRef]

40. Jin, W.; Li, X.; Wan, B. A Highly diastereo-and enantioselective copper(I)-catalyzed Henry reaction using a bis (sulfonamide)diamine ligand. J. Org. Chem. 2011, 76, 484-491. [CrossRef] [PubMed]

41. Chunhong, Z.; Liu, F.; Gou, S. Application of chiral N, N'-dialkyl-1, 2-cyclohexanediamine derivatives in asymmetric copper(II)catalyzed Henry reactions. Tetrahedron Asymmetry 2014, 25, 278-283. [CrossRef]

42. Khlebnikova, T.B.; Konev, V.N.; Pai, Z.P. Levopimaric acid derived 1, 2-diamines and their application in the copper-catalyzed asymmetric henry reaction. Tetrahedron 2018, 74, 260-267. [CrossRef]

43. Lu, G.; Zheng, F.; Wang, L.; Guo, Y.; Li, X.; Cao, X.; Wang, C.; Chi, H.; Dong, Y.; Zhang, Z. Asymmetric henry reaction catalyzed by cu (ii)-based chiral amino alcohol complexes with c2-symmetry. Tetrahedron Asymmetry 2016, 27, 732-739. [CrossRef]

44. Shibasaki, M.; Kanai, M.; Gröger, H. Nitroaldol reaction. In Comprehensive Asymmetric Catalysis: Supplement; Springer: Berlin/Heidelberg, Germany, 2004; Volume 1. 
45. Palomo, C.; Oiarbide, M.; Mielgo, A. Unveiling reliable catalysts for the asymmetric nitroaldol (Henry) reaction. Angew. Chem. Int. Ed. 2004, 43, 5442-5444.

46. Boruwa, J.; Gogoi, N.; Saikia, P.P.; Barua, N.C. Catalytic asymmetric Henry reaction. Tetrahedron Asymmetry 2006, 17, 3315-3326. [CrossRef]

47. Palomo, C.; Oiarbide, M.; Laso, A. Recent advances in the catalytic asymmetric nitroaldol (Henry) reaction. Eur. J. Org. Chem. 2007, 2007, 2561-2574. [CrossRef]

48. Blay, G.; Hernandez-Olmos, V.; Pedro, J.R. Development of new N, N-ligands for the enantioselective copper(II)-catalyzed Henry reaction. Synlett 2011, 2011, 1195-1211. [CrossRef]

49. Shashank, A.B.; Ramachary, D.B. Organocatalytic diastereoselective synthesis of chiral decalines through the domino ClaisenSchmidt/Henry reaction. Org. Biomol. Chem. 2015, 13, 5110-5114. [CrossRef]

50. Knudsen, K.R.; Risgaard, T.; Nishiwaki, N.; Gothelf, K.V.; Jørgensen, K.A. The first catalytic asymmetric aza-Henry reaction of nitronates with imines: A novel approach to optically active $\beta$-nitro- $\alpha$-amino acid-and $\alpha$, $\beta$-diamino acid derivatives. J. Am. Chem. Soc. 2001, 123, 5843-5844. [CrossRef] [PubMed]

51. Kogami, Y.; Nakajima, T.; Ikeno, T.; Yamada, T. Enantioselective Henry reaction catalyzed by salen-cobalt complexes. Synthesis 2004, 2004, 1947-1950. [CrossRef]

52. Palomo, C.; Oiarbide, M.; Laso, A. Enantioselective Henry reactions under dual Lewis acid/amine catalysis using chiral amino alcohol ligands. Angew. Chem. Int. Ed. 2005, 44, 3881-3884. [CrossRef]

53. Farina, V.; Reeves, J.T.; Senanayake, C.H.; Song, J.J. Asymmetric synthesis of active pharmaceutical ingredients. Chem. Rev. 2006, 106, 2734-2793. [CrossRef]

54. Mellah, M.; Voituriez, A.; Schulz, E. Chiral sulfur ligands for asymmetric catalysis. Chem. Rev. 2007, 107, 5133-5209. [CrossRef]

55. Trippé, G.; Canevet, D.; Le Derf, F.; Frère, P.; Sallé, M. An extended tetrathiafulvalene redox-ligand incorporating a thiophene spacer. Tetrahedron Lett. 2008, 49, 5452-5454. [CrossRef]

56. Jimenez, R.P.; Parvez, M.; Sutherland, T.C.; Viccars, J. Synthesis and Optical and Electronic Properties of Thiophene Derivatives; Wiley Online Library: Hoboken, NJ, USA, 2009.

57. Chen, X.; Elsenbaumer, R.L. Synthesis of thieno [3, 4-d]-1, 3-dithiol-2-one derivatives. Tetrahedron Lett. 2009, 50, 3750-3752 [CrossRef]

58. Ley, S.V.; Smith, S.C.; Woodward, P.R. Further reactions of t-butyl 3-oxobutanthioate and t-butyl 4-diethyl-phosphono-3oxobutanthioate: Carbonyl coupling reactions, amination, use in the preparation of 3-acyltetramic acids and application to the total synthesis of fuligorubin A. Tetrahedron 1992, 48, 1145-1174. [CrossRef]

59. Fondo, M.; Corredoira-Vázquez, J.; GarcíaDeibe, A.M.; Sanmartín-Matalobos, J. An easy approach to obtain alcohol-amines by reduction of alcohol functionalized imines. Proceedings 2019, 9, 2. [CrossRef]

60. Sreenath, K.; Yuan, Z.; Macias-Contreras, M.; Ramachandran, V.; Clark, R.J.; Zhu, L. Dual role of acetate in copper (II) acetate catalyzed dehydrogenation of chelating aromatic secondary amines: A kinetic case study of copper-catalyzed oxidation reactions. Eur. J. Inorg. Chem. 2016, 2016, 3728-3743. [CrossRef]

61. Wang, Z.; Wu, X.; Li, Z.; Huang, Z.; Chen, F. Ketoreductase catalyzed stereoselective bioreduction of $\alpha$-nitro ketones. Org. Biomol. Chem. 2019, 17, 3575-3580. [CrossRef] [PubMed]

62. Qin, D.D.; Lai, W.H.; Hu, D.; Chen, Z.; Wu, A.A.; Ruan, Y.P.; Zhou, Z.H.; Chen, H.B. Highly enantioselective henry reactions of aromatic aldehydes catalyzed by an amino alcohol-copper(II) complex. Chem. Eur. J. 2012, 18, 10515-10518. [CrossRef] [PubMed] 